\title{
Comparing geometrical and delay radio emission heights in pulsars
}

\author{
D. Mitra ${ }^{1}$ and X. H. $\mathrm{Li}^{1,2}$ \\ 1 Max-Planck Institute für Radioastronomie, Auf dem Hügel 69, 53121, Bonn, Germany \\ 2 National Astronomical Observatory, Chinese Academy of Sciences, Jia-20 DaTun Road, Chaoyang District, \\ Beijing 100012, PR China
}

Received 18 July 2003 / Accepted 18 December 2003

\begin{abstract}
We use a set of carefully selected, published average multifrequency polarimetric observations for six bright cone-dominated pulsars and devise a method to combine the multifrequency polarization position angle (PPA) sweep traverses. We demonstrate that the PPA traverse is in excellent agreement with the rotating vector model over this broad frequency range, confirming that radio emission emanates from perfectly dipolar field lines. Correcting for the effect of retardation we firmly establish the steepest gradient point in the combined PPA traverse to be the fiducial phase in these pulsars. We use this combined curve and inputs from earlier studies to determine the geometrical angles of the neutron star, namely $\alpha$ - the angle between the rotation and the dipole magnetic axis and $\beta$ - the angle between the magnetic axis and the observers line of sight. Using these estimates of $\alpha$ and $\beta$ we derive the geometrical emission heights $\left(r_{\text {geo }}\right)$. Further, using the relativistic beaming model based on effects of aberration and retardation, we find the delay emission heights $r_{\text {delay }}^{\text {BCW }}$ suggested by Blaskiewicz et al. (1991). We find in general $r_{\text {delay }}^{\mathrm{BCW}}<r_{\text {geo }}$, which can be explained by a broad emission region operating in pulsars or/and the signature of the magnetic field sweepback effect as suggested by Dyks et al. (2003).

For pulsars with central core emission in our sample, we find the peak of the central core component to lag behind the steepest gradient of the PPA traverse at several frequencies. Significant frequency evolution of the core width is observed over this frequency range. The above facts strongly suggest: (a) the peak core emission does not lie on the fiducial plane containing the dipole magnetic axis and the rotation axis, and (b) the core emission does not originate from the polar cap surface.
\end{abstract}

Key words. stars: pulsars: individual: PSR B0301+19, PSR B0525+21, PSR B1039-19, PSR B1737+13, PSR B2045-16, PSR B2111+46

\section{Introduction}

Pulsars exhibit broadband radio emission ranging over tens of $\mathrm{MHz}$ to tens of $\mathrm{GHz}$ and the emission is highly polarized. Inspection of pulse shapes at multiple frequencies suggests that in general the pulse radio emission beam consists of nested cones of emission, and a central core emission (Mitra \& Deshpande 1999). It is most often seen that pulse widths at low observable radio frequencies are wider than that at higher frequencies. This observation, commonly referred to as radius to frequency mapping (RFM), has been interpreted as low frequency emission arising at higher altitude from the surface of the neutron star while higher frequencies arise closer to the stellar surface (Cordes 1978).

Radio emission from pulsars is thought to arise from dipolar magnetic field lines. Evidence for this underlying magnetic field comes from the observed "S-shaped" swing of the polarization position angle (PPA) across the pulse. To interpret this observed swing, Radhakrishnan \& Cooke (1969)

Send offprint requests to: $\mathrm{D}$. Mitra, e-mail: dmitra@mpifr-bonn.mpg.de proposed the rotating vector model (RVM) which is interpreted in terms of radiation being beamed along the open dipolar field lines and the plane of linear polarization is that containing the field line associated the emission received at a given instant. According to the model the PPA $\psi$ across the pulse phase $\phi$ can be represented as

$\psi=\psi_{0}+\arctan \left(\frac{\sin \alpha \sin \left(\phi-\phi_{0}\right)}{\sin \xi \cos \alpha-\sin \alpha \cos \xi \cos \left(\phi-\phi_{0}\right)}\right)$.

(Manchester \& Taylor 1977). Here $\alpha$ is the angle between the rotation axis and the magnetic axis and $\beta$ is the angle between the rotation axis and the line of sight to the pulsar. $\psi_{0}$ and $\phi_{0}$ are the PPA and phase offset of the observed curve respectively. The angle $\xi=\alpha+\beta$ is the angle between the observer's line of sight and the pulsar rotation axis. While the RVM model has been successfully applied to a large number of pulsars, for several of them the PPA traverse seem to show significant distortions. However even for pulsars where the PPA traverses are in agreement with the RVM, accurate determination of $\alpha$ and $\beta$ is extremely difficult (Narayan \& Vivekanand 1982; von Hoensbroech \& Xilouris 1997, HX97a 
Table 1. The pulsar parameters used in our analysis. Columns 1-3 gives the PSR Bname, Period and the morphological type classified by Rankin (1993a). Columns $4-6$ gives the values of $\alpha, \beta$ and reduced $\chi^{2}$ obtained by us (indicated as our) after the combined fits. Columns $7-10$ give the $\alpha$ and $\beta$ from Lyne \& Manchester (1988, indicated as LM88) and Rankin (1993b, indicated as R93). The superscript dagger corresponding to these $\alpha$ values are cases where one needs to do the transformation $(180-\alpha)$ to compare with our values. Columns 11 gives the value of the opening angle of the polar cap $\rho_{\mathrm{pc}}$.

\begin{tabular}{|c|c|c|c|c|c|c|c|c|c|c|}
\hline PSR Bname & Period (s) & CLASS & $\begin{array}{l}\alpha\left(^{\circ}\right) \\
\text { (our) }\end{array}$ & $\begin{array}{l}\beta\left(^{\circ}\right) \\
\text { (our) }\end{array}$ & $x^{2}$ & $\begin{array}{l}\alpha\left(^{\circ}\right) \\
\text { R93 }\end{array}$ & $\begin{array}{l}\beta\left(^{\circ}\right) \\
\text { R93 }\end{array}$ & $\begin{array}{r}\alpha\left(^{\circ}\right) \\
\text { LM } 88\end{array}$ & $\begin{array}{r}\beta\left(^{\circ}\right) \\
\text { LM88 }\end{array}$ & $\begin{array}{c}\rho_{\mathrm{pc}} \\
\left({ }^{\circ}\right)\end{array}$ \\
\hline В0301+19 & 1.387 & $\mathrm{D}$ & $159 \pm 16$ & $-1.1 \pm 0.5$ & 2.6 & $31.9^{\dagger}$ & 1.8 & $30^{\dagger}$ & 1.7 & $1.05 \pm 0.3$ \\
\hline B $0525+21$ & 3.745 & $\mathrm{D}$ & $54 \pm 4$ & $1.3 \pm 0.1$ & 4.1 & 23.2 & 0.7 & 21 & 0.6 & $0.64 \pm 0.2$ \\
\hline B1039-19 & 1.386 & M & $51 \pm 19$ & $2.5 \pm 0.3$ & 2.5 & 33.8 & 1.8 & 31 & 1.7 & $1.05 \pm 0.3$ \\
\hline B $1737+13$ & 0.803 & M & $47 \pm 12$ & $2.1 \pm 0.4$ & 5.6 & 40.8 & 2.5 & 41 & 1.9 & $1.38 \pm 0.4$ \\
\hline B2045-16 & 1.961 & $\mathrm{~T}$ & $127 \pm 2$ & $-1.4 \pm 0.1$ & 12.1 & $36.7^{\dagger}$ & 1.1 & $36^{\dagger}$ & 1.1 & $0.88 \pm 0.3$ \\
\hline B2111+46 & 1.014 & $\mathrm{~T}$ & $14 \pm 4$ & $-1.4 \pm 0.5$ & 21.3 & 8.6 & 1.3 & 9 & 1.4 & $1.23 \pm 0.4$ \\
\hline
\end{tabular}

hereafter). Rankin (1990) suggested another method to estimate $\alpha$ for core-dominated pulsars. She noticed that the $3 \mathrm{~dB}$ core widths at $1 \mathrm{GHz}$ is related to $\alpha$ and the pulsar pe$\operatorname{riod} P$ as $2.45^{\circ} P^{-0.5} / \sin \alpha$. This angular width corresponds roughly to the size of the polar cap and thus implies that the core emission should originate from the polar cap. Further, the RVM model can be used to calculate $\beta$. However there has been the suggestion that the core emission might originate far from the stellar surface (Gil 1991), thus making it conceptually difficult to use this method.

Accepting that the geometrical angles of the star can be determined by measuring the pulse width, the geometrical emission height $r_{\text {geo }}$ can be found (see Mitra \& Rankin 2002, hereafter MR02 for a recent review). Thus, pulse width measurements are based on two arbitrarily defined points on the leading and trailing edge of the profile. It is currently impossible to determine the field line with respect to the last open field line for these defined points. This uncertainty causes difficultly in our understanding of what the emission height $r_{\text {geo }}$ might be corresponding to. We will come back to this important question in a later section of this paper.

The RVM model was further advanced by Blaskiewicz et al. (1991, hereafter BCW) where they included first order special relativistic effects. The model predicts that due to aberration and retardation $(\mathrm{A} / \mathrm{R})$ effects the steepest gradient point of the PPA traverse has a time lag with respect to the center of the total intensity pulse profile. This time lag can be converted to an emission height $r_{\text {delay }}^{\mathrm{BCW}}$. Indeed, observation confirms that for $95 \%$ of the cases subject to such study, the predicted lag is seen (BCW, HX97a). Hibschman \& Arons (2001) repeated the analysis of $\mathrm{A} / \mathrm{R}$ effects and confirmed the $\mathrm{BCW}$ results. Moreover the time lag seems to increase with decreasing frequency as predicted by RFM (BCW, HX97a and Malov \& Suleimanova 1998). This method of estimating the emission heights has the advantage that it is independent of the geometrical parameters $\alpha$ and $\beta$. However this technique cannot be reliably applied to several pulsars showing distorted PPA traverse where one encounters difficulties in establishing the steepest gradient point in the curve (a detailed discussion on this can be found in HX97a).

Recently, a series of paper by Gangadhara \& Gupta (2001) and Gupta \& Gangadhara (2003) (GGa and GGb hereafter) has applied the $\mathrm{A} / \mathrm{R}$ theory to total intensity profiles in pulsars and estimated emission heights $r_{\text {delay }}^{\mathrm{GGa}}$. Although the formula derived by GGa depend on $\alpha$ and $\beta$, recently Dyks et al. (2003) revisited the problem and have shown that $r_{\text {delay }}^{\mathrm{GG}}$ estimates are also independent of the geometrical angles, just as the BCW method.

While there is no clear consensus, the mentioned above methods seem to support the fact that RFM exists in pulsars. Theoretical models for pulsar emission predict no RFM where the spectral index $a$ is 0 (Barnard \& Arons 1986) to a situation where $a$ is 0.66 (Ruderman \& Sutherland 1975). Also observationally this wide variety is seen (Kijak \& Gil 1997; MR02; HX97a). However it is often seen that the estimated heights $r_{\text {geo }}$ and $r_{\text {delay }}$ are in disagreement (BCW). Comparison of these height estimates are often difficult due to the enormous uncertainties involved in estimating $\alpha$ and $\beta$. In this work, we use a set of six strong conal pulsars to do a systematic analysis of RFM in detail. The selected data set consists of multifrequency polarimetric data from Gould \& Lyne (1998) and von Hoensbroech \& Xilouris 1997 (HX97b) and is discussed in Sect. 2. We devise a method to combine PPA traverse at multiple frequencies and find the corresponding $\alpha$ and $\beta$ as discussed in Sect. 3. In Sect. 4 we investigate the inter-relationship between the improved $r_{\text {geo }}$ and $r_{\text {delay }}$. In Sect. 5 we summarize our results.

\section{Data selection}

Table 1 lists the pulsars we use for our analysis. Pulsar choice is based on the criteria: (1) they have smoothly varying PPA traverse observed over a frequency range of $0.4-4.85 \mathrm{GHz}$, and (2) all the pulsars show clear evidence of outer conal components over this frequency range. Using these criteria we selected six strong pulsars for which multifrequency polarimetric data was available from the European Pulsar Network (EPN) archive maintained by the Max-Planck Institut für Radioastronomie, Bonn ${ }^{1}$. We used published data at 0.4, 0.6, 0.9, 1.4 and 1.6 GHz from Gould \& Lyne (1998) and at $4.85 \mathrm{GHz}$ from HX97b. In total we use 31 profiles spaced over this frequency range. The sample of pulsars belong to the morphological class of conal double (D), triple (T) and

\footnotetext{
${ }^{1}$ http://www.mpifr-bonn.mpg.de/div/pulsar/data/
} 
multiple (M) as classified by Rankin (1993) and mentioned in Table 1.

Three pulsars in our sample, namely PSR B1737+17, PSR B2045-16 and PSR B2111+46, have clearly identifiable core components. Classification of the core component is based on the sign changing circular polarization observed in average profiles as suggested by Rankin (1990). A sign changing circular polarization is also seen for PSR B1039-19 in the leading part of the profile, however as suggested by Han et al. (1998) in several complex profiles such sign changing circular polarization is not only restricted to the core component. Based on the location of this component, it seems that this feature is not associated with the central core emission. Further multifrequency Gaussian fits (see Kramer 1994) to the profiles shows that 4 or 5 components fit the pulse shape well for this pulsar, thus making PSR B1039-19 more a M type pulsar.

The full Stokes $(I, Q, U, V)$ parameter data is used to construct the PPA, $\psi=0.5 \tan ^{-1}(U / Q)$. The linear polarization is first calculated as $\sqrt{U^{2}+Q^{2}}$ and then the off-pulse mean is subtracted to remove the positive bias. PPA points for which the linear polarization exceeds 3 times the off-pulse noise of the linear polarization is used in our analysis. The error on $\psi$ is calculated as $\sigma_{\psi}=\sqrt{\left(Q \sigma_{Q}\right)^{2}+\left(U \sigma_{U}\right)^{2}} / 2 L^{2}$, where $\sigma_{Q}$ and $\sigma_{U}$ are the off pulse rms in Stokes $Q$ and $U$ respectively. It has been recently shown by Li \& Han (2003) that the PPA traverse can be affected strongly by interstellar scattering. We note that none of the pulsars in our sample show any scattering, and thus the PPA traverse mostly reflects intrinsic properties within the pulsar magnetosphere.

\section{Determining the viewing geometry}

If RVM is assumed, then the PPA traverse observed at several frequencies should be the same as the viewing geometry is constant for a given star (see Eq. (1)). The only difference is that with decreasing frequency the overall broadening of the PPA traverse will extend over wider longitude ranges. Thus in principal fitting an RVM to the lowest observable frequency should give the best solution for the viewing geometry. On the other hand for, most of the conal double profiles (e.g. PSR B0525+21) the linear polarization towards the center of the profile is low and thus the PPA has large uncertainties which is more prominently seen at lower frequencies. Thus to fill the PPA traverse for these central longitudes the high frequency profiles are helpful. Hence it is desirable to combine all the available frequencies and fit the RVM to the combined PPA traverse to obtain better estimates of $\alpha$ and $\beta$. The pulse widths need to be as large as $\sim 50^{\circ}$ to be able to improve estimates of $\alpha$ and $\beta$ significantly for this reason.

According to the $\mathrm{BCW}$ model, the behaviour of the PPA traverse depends on the emission height and the pulsar period (see Eq. (16) in BCW). This A/R effect is enhanced in millisecond pulsars (Gil \& Krawczyk 1997) where the pulsar rotates significantly faster. While for normal slower pulsars such as in our sample, BCW already note that a phase-shifted RVM fits the PPA traverse reasonably well. A similar conclusion has also been reached by Hibschman \& Arons (2001). Considering this to be the premise for interpreting the multifrequency
PPA traverse, in the rest of this section we outline the ways in which we are able to combine the multifrequency PPA traverse and estimate the viewing geometry.

\subsection{Earlier studies dealing with viewing geometry}

The prime difficulty encountered while fitting the RVM given by Eq. (1) to the PPA swing curve is that several combination of $\alpha$ and $\beta$ can produce equally acceptable fits (see HX97a). Further, Narayan \& Vivekanand (1982) pointed out that to constrain these angles better one needs the PPA traverse to extend over wide longitude ranges and particularly that the only way to discriminate if the line of sight passes outside (outer) or inside (inner) the rotation axis and the magnetic axis is to have wider profiles. A detailed description of the RVM fitting proccedure and definitions can be found in Everett \& Weisberg (2001, hereafter EW).

This work of EW reviews the various methods that have been used to obtain the viewing geometry in the past and further fits RVM to several pulsars using a rigorous treatment of statistical errors on PPAs. To include sufficient number of points in the profile wings they considered points for which the signal to noise ratio (SNR) of the unbiased linear polarization exceeds 1.57. This way PPA points are retained at longitude ranges where there is no pulsed emission. In comparison, we have considered points with SNR greater than 3, and thus are certain that lower SNR points are not affecting the fits.

Nonetheless the covariant nature of $\alpha$ and $\beta$ makes it impossible to constrain the viewing geometry. The study of Lyne \& Manchester (1988) and Rankin $(1990,1993)$ is of great importance, as they have tried to use the profile width information (which is more sensitive to $\alpha$ than $\beta$ ) along with the steepest gradient of the PPA traverse to constrain the viewing geometry (see EW for detailed description). The most remarkable aspect of the above two studies is that from two different set of arguments they obtain very similar values of $\alpha$ and $\beta$. Hence we have used their values as initial guesses while fitting the PPA traverse. Thus here we have two major goals: (a) to examine the validity of RVM over a wide frequency range; (b) to assess if RFM can obtain wider PPAs near the wings of the profile enabling better estimation of the viewing geometry.

\subsection{The problem of PPA moding}

EW noticed that several average RVM-style PPA traverse, on close inspection tend to be slightly incorrect. A possible reason for this is that pulsar radiation is often in the form of two orthogonal modes, and mixing of these modes at some longitude ranges in the pulse can corrupt the average classical RVM. Such orthogonal as well as non-orthogonal moding has been observed in several pulsars. There is a danger thus in using the average PPA traverse to obtain pulsar parameters. Most of the pulsars considered follow the RVM reasonably well. There are two pulsars in our sample, PSR B0301+19 and B0525+21, where comparison of single pulse and average profiles are available (Backer \& Rankin 1980; Stinebring et al. 1984). For both cases the average PPA is in good agreement with the single 
pulse PPA. Only towards the wings of the profiles are some low level modal effects seen (see Rankin \& Ramachandran 2003 for discussion). Strong modal effects should suggest phase dependent distortion of the PPA traverse, and are known to vary with frequency (Karastergiou et al. 2002). Our sample pulsars however are free from such distortions.

\subsection{Combining the multifrequency PPA traverse}

As a first step we fit the RVM given by Eq. (1) to a given pulsar at every available frequency. The fits are done using the method outlined in HX97a to obtain the best fit values for $\alpha, \beta, \phi_{0}$ and $\psi_{0}$. The method uses the Levenberg-Marquart Algorithm (Marquart 1963) as implemented in Numerical recipes (Press et al. 1986) and is sensitive to the initial parameters. As initial inputs we used $\alpha$ and $\beta$ values from Rankin (1993). The values obtained by Rankin (1993) and Lyne \& Manchester (1988) are listed in Table 1 and are very similar. This unconstrained fit resulted in highly correlated values of $\alpha$ and $\beta$. The formal $1 \sigma$ errors in $\alpha, \beta \psi_{0}$ and $\phi_{0}$ are obtained from the $\chi^{2}$ fitting procedure using the resultant covariance matrix as prescribed in Press et al. (1986). The phase of the steepest gradient point $\phi_{0}$ in the RVM fit is found by zeroing the second derivative of Eq. (1) with respect to the pulse phase $\phi$ as discussed in HX97a. The values are found to be in good agreement with that obtained from the formal fitting procedure.

In Cols. $3-5$ of Table 2 the estimated $\alpha, \beta$ and the reduced $\chi^{2}$ values for all pulsars at various frequencies are given. The steepest gradient of the PPA traverse $(\partial \psi / \partial \phi)_{\max }=\sin \alpha / \sin \beta$ is tabulated in Col. 6. The RVM fits are in excellent agreement for each pulsar over this wide frequency range. Thus we are now in a position to combine the PPA traverse. We do this firstly by subtracting the offsets $\phi_{0}$ and $\psi_{0}$ of the PPA traverse at each frequency and then overlapping them. The combined PPA traverse for all the pulsars are shown in Fig. 1. This combined PPA traverses are now fitted to obtain $\alpha$ and $\beta$. These values eventually obtained have significantly smaller error bars than the individual fits. However $\alpha$ and $\beta$ still remain highly correlated. Note that the reduced $\chi^{2}$ value for PSR B2045-16 and $\mathrm{B} 2111+46$ are somewhat large, which is due to the 4.85 GHz PPA traverse showing larger deviation from the other frequencies. However for 5 of the cases the profile widths are not wide enough to find if the lines of sight are inner or outer. Only for PSR B2111+46 is the line of sight inner and consistent with the findings of Athanasiadis et al. (2003). It is noteworthy that we have obtained convergent fits for PSR B0301+19, B0525+21 and B2045-16. For all these pulsars different inputs of $\alpha$ and $\beta$ obtained from previous studies converged to the same solution.

The smaller error estimates of $\alpha$ and $\beta$ are possible primarily due to (a) large number of independent points available in the combined PPA to fit the RVM and (b) RFM enabling parts of the PPA traverse to be filled by independent measurements. The estimated error reflects the covariant relationship between these parameters. Unfortunately there is little improvement between the nature of correlation between $\alpha$ and $\beta$ for this combined PPA traverse compared to the curves at individual frequency. For comparison we show by a dashed curve in the bottom panel of Fig. 1 for which $\alpha$ and $\beta$ are used from Rankin (1993b). For most of the cases the solid and the dashed lines are indistinguisable reflecting the highly correlation between $\alpha$ and $\beta$. Only for PSR B $0525+21$ is our fitted line (solid) better than the literature value. The reason for this high correlation is that most of the pulsars in our sample have widths of $\sim 20^{\circ}$, not sensitive enough to affect the fits. The increase in width is only marginal, typically a factor of 1.3 between the highest to lowest frequency as seen in Cols. 7-9 in Table 2. Thus to obtain better estimates of the viewing geometry, low frequency polarimetric observations are absolutely essential where due to RFM wider profiles are observed.

The agreement of the combined PPA traverses with the RVM over this wide frequency range can be used to identify the fiducial phases for these pulsars. The fiducial phase is defined as the point lying in the plane containing the dipole magnetic axis, the rotation axis and the observers line of sight. The steepest gradient point in the PPA traverse is identified as such a point according to RVM. Due to the A/R effects proposed by the BCW model the steepest gradient point is retarded towards later longitudes by an amount proportional to $r(v) / c$ in a frequency $(v)$ dependent manner, where $r(v)$ is the emission height and $c$ is the velocity of light. In the process of producing combined PPA traverses we have eliminated the effect of retardation. Given the excellent symmetry of the combined PPA traverses observed for all the pulsars we conclude that the inflexion or the steepest gradient point undoubtedly is the location of the fiducial phase in these pulsars. This fiducial phase should be used for cold plasma dispersion correction and timing analysis in pulsars.

\section{Comparing radio emission heights}

There exist two methods widely used to obtain radio emission heights in pulsars. In this section we mention briefly these methods and then compare the emission heights for our sample of pulsars.

\subsection{Emission heigths using the geometrical method: $r_{\text {geo }}$}

Assuming emission arising from open dipolar field lines and a circular emission beam shape, the geometrical emission height $r_{\text {geo }}$ can be found. The formula (see Kijak \& Gil 1997) is,

$r_{\mathrm{geo}} \simeq R_{*} P\left(\frac{\rho}{1.23}\right)^{2} \mathrm{~s}^{-2} \mathrm{~km}$.

Here $\rho$ is the radius of the emission beam calculated as $\sin ^{2}(\rho / 2)=\sin (\alpha+\beta) \sin (\alpha) \sin ^{2}(W / 4)+\sin ^{2}(\beta / 2)$, (Gil et al. 1984). $P$ is the period of the pulsar in seconds and $W$ is the measured pulse width. The parameter $s$ lying between $0 \leq s \leq 1$ describes the locus of the field line at the polar cap, where $s=0$ at the magnetic pole and $s=1$ at the edge of the Goldreich-Julian (1969) circular polar cap and the radius $R_{*}$ is $10 \mathrm{~km}$. Before estimating $r_{\text {geo }}$ for our sample of pulsars, we first review the factors affecting determination of $r_{\text {geo }}$. 
PSR B0301+19

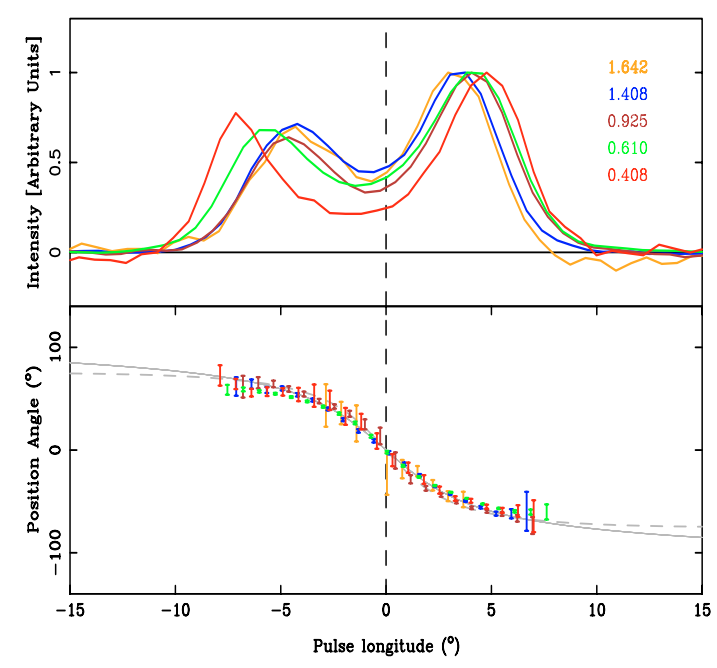

PSR B0525+21

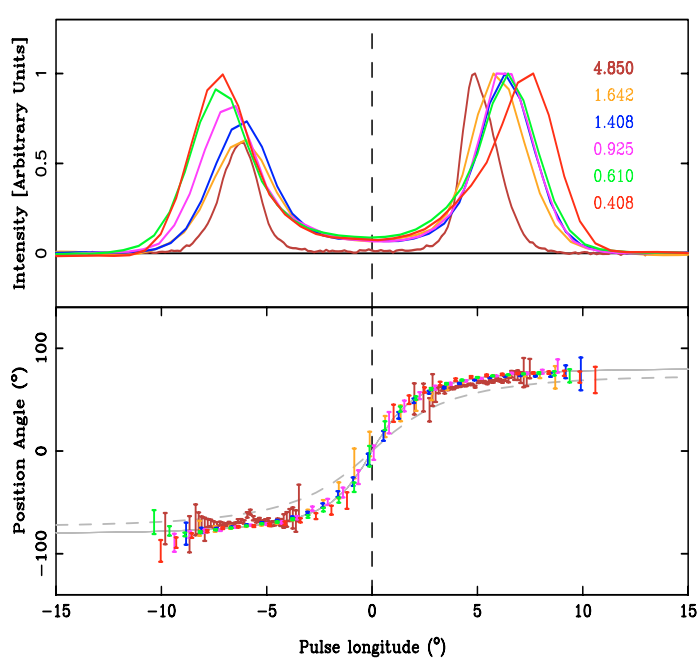

PSR B1039-19

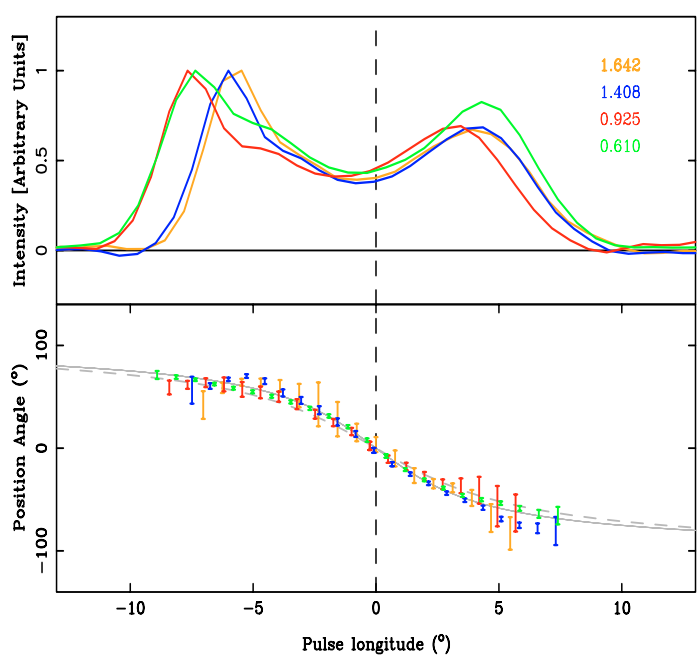

PSR B1737+13

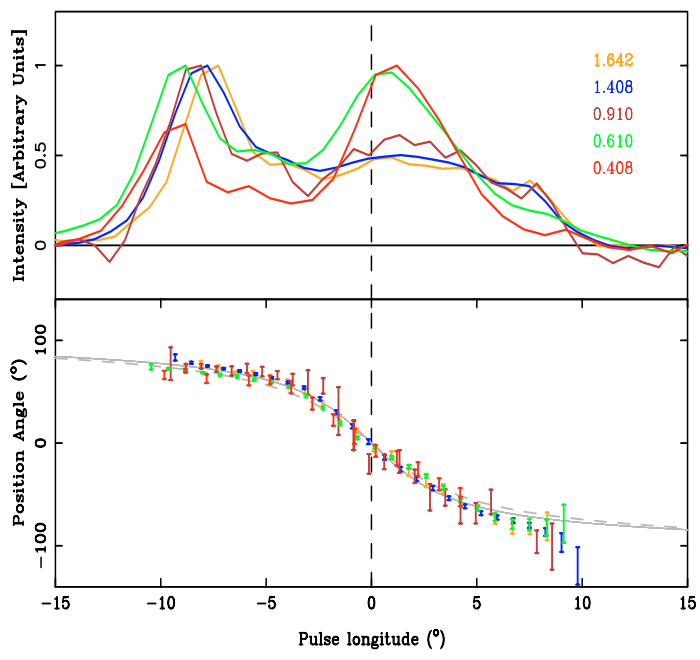

PSR B2045-16

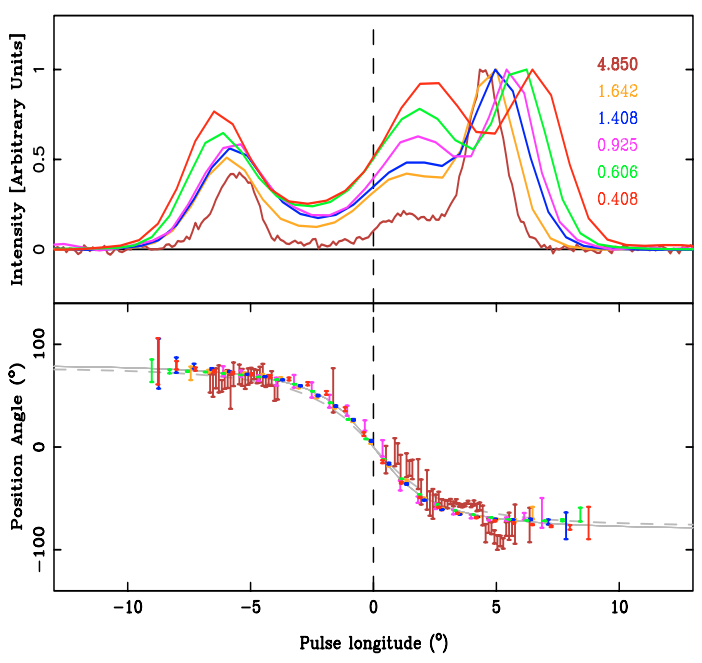

PSR B2111+46

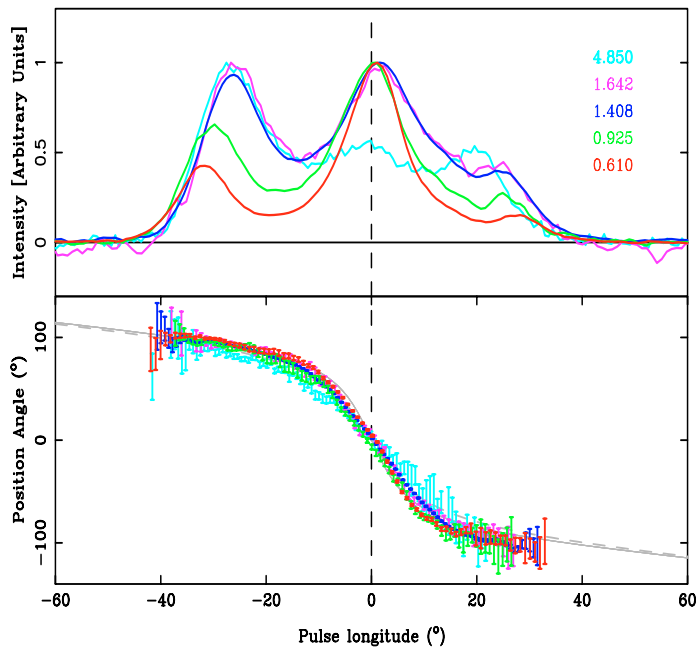

Fig. 1. The profiles aligned with respect to the center of the PPA traverse at various frequencies. In the top panel the total intensity of the profiles are plotted by lines with different colours. The colour coding used for every frequency is indicated in the top panel. The bottom panel show the combined PPA traverse and the same symbol coding is used. The central $x=0$ line is indicated by the dashed line in both panel. The solid gray line is the best fit RVM to the combined PPA traverse and the dashed line corresponds to RVM using values of the viewing geometry from Rankin (1993). 
Table 2. The angles $\alpha, \beta$ are fits obtained from the RVM model at every frequency and the $\chi^{2}$ for the fits are given. The steepest gradient is designated by $\left(\frac{\mathrm{d} \psi}{\mathrm{d} \phi}\right)_{\max }$. The various widths $(W)$ and the geometrical radius $r_{\text {geo }}$ estimates for the OTS, OTHX and PPCS points are tabulated.

\begin{tabular}{|c|c|c|c|c|c|c|c|c|c|c|c|}
\hline PSR B & $\begin{array}{l}\text { Freq. } \\
(\mathrm{GHz})\end{array}$ & $\begin{array}{c}\alpha \\
\left({ }^{\circ}\right)\end{array}$ & $\begin{array}{c}\beta \\
\left({ }^{\circ}\right)\end{array}$ & $\chi^{2}$ & $\left(\frac{\mathrm{d} \psi}{\mathrm{d} \phi}\right)_{\max }$ & $\begin{array}{r}W_{\text {OTS }} \\
\left(^{\circ}\right)\end{array}$ & $\begin{array}{c}W_{\text {ОтнХ }} \\
\left(^{\circ}\right)\end{array}$ & $\begin{array}{r}W_{\text {PPCS }} \\
\quad\left(^{\circ}\right)\end{array}$ & $\begin{array}{l}r_{\text {geo }}^{\text {OTS }} \\
(\mathrm{km})\end{array}$ & $\begin{array}{l}r_{\text {geo }}^{\text {OTHX }} \\
(\mathrm{km})\end{array}$ & $\begin{array}{l}r_{\mathrm{geo}}^{\mathrm{PPCS}} \\
(\mathrm{km})\end{array}$ \\
\hline \multirow[t]{5}{*}{ B $0301+19$} & 0.408 & $168 \pm 299$ & $-0.6 \pm 14.0$ & 0.4 & $-18 \pm 28$ & $18.9 \pm 0.5$ & $18.5 \pm 0.5$ & $11.57 \pm 0.04$ & $122 \pm 159$ & $118 \pm 153$ & $53 \pm 60$ \\
\hline & 0.610 & $166 \pm 63$ & $-0.7 \pm 3.4$ & 3.2 & $-17 \pm 9$ & $22.9 \pm 0.7$ & $22.0 \pm 0.7$ & $9.76 \pm 0.01$ & $174 \pm 233$ & $162 \pm 215$ & $41 \pm 44$ \\
\hline & 0.925 & $158 \pm 106$ & $-1.0 \pm 4.5$ & 0.1 & $-23 \pm 24$ & $19.2 \pm 0.3$ & $18.7 \pm 0.3$ & $8.73 \pm 0.01$ & $126 \pm 164$ & $120 \pm 156$ & $34 \pm 36$ \\
\hline & 1.408 & $133 \pm 49$ & $-2.3 \pm 1.9$ & 1.3 & $-18 \pm 12$ & $19.0 \pm 0.3$ & $18.8 \pm 0.3$ & $7.91 \pm 0.01$ & $124 \pm 162$ & $121 \pm 157$ & $31 \pm 30$ \\
\hline & 1.642 & $158 \pm 2323$ & $-1.0 \pm 118$ & 0.1 & $-23 \pm 408$ & $15.1 \pm 0.6$ & $14.8 \pm 0.6$ & $7.62 \pm 0.04$ & $82 \pm 101$ & $79 \pm 98$ & $29 \pm 28$ \\
\hline \multirow[t]{6}{*}{ B $0525+21$} & 0.408 & $14 \pm 38$ & $0.3 \pm 0.8$ & 1.8 & $41 \pm 15$ & $21.4 \pm 0.3$ & $21.4 \pm 0.3$ & $14.61 \pm 0.01$ & $1946 \pm 616$ & $1946 \pm 615$ & $928 \pm 292$ \\
\hline & 0.610 & $47 \pm 7$ & $1.1 \pm 0.2$ & 1.1 & $37 \pm 7$ & $23.2 \pm 0.5$ & $23.1 \pm 0.5$ & $13.73 \pm 0.01$ & $2269 \pm 722$ & $2247 \pm 715$ & $825 \pm 259$ \\
\hline & 0.925 & $81 \pm 9$ & $1.7 \pm 0.1$ & 0.6 & $33 \pm 2$ & $21.2 \pm 0.3$ & $21.1 \pm 0.2$ & $13.19 \pm 0.01$ & $1909 \pm 605$ & $1880 \pm 594$ & $764 \pm 240$ \\
\hline & 1.408 & $40 \pm 10$ & $1.1 \pm 0.3$ & 0.5 & $33 \pm 10$ & $22.0 \pm 0.3$ & $21.7 \pm 0.3$ & $12.52 \pm 0.01$ & $2049 \pm 649$ & $2005 \pm 634$ & $693 \pm 217$ \\
\hline & 1.642 & $73 \pm 17$ & $1.6 \pm 0.2$ & 0.5 & $33 \pm 5$ & $21.4 \pm 0.5$ & $20.7 \pm 0.5$ & $12.11 \pm 0.01$ & $1953 \pm 622$ & $1819 \pm 579$ & $651 \pm 205$ \\
\hline & 4.850 & $50 \pm 21$ & $1.5 \pm 0.5$ & 1.3 & $29 \pm 13$ & $18.2 \pm 0.2$ & $17.8 \pm 0.1$ & $11.13 \pm 0.01$ & $1413 \pm 447$ & $1316 \pm 431$ & $556 \pm 175$ \\
\hline \multirow[t]{4}{*}{ B1039-19 } & 0.610 & $50 \pm 32$ & $-2.7 \pm 1.2$ & 0.7 & $-16 \pm 1$ & $20.4 \pm 0.4$ & $20.3 \pm 0.4$ & $11.62 \pm 0.02$ & $658 \pm 379$ & $650 \pm 374$ & $252 \pm 130$ \\
\hline & 0.925 & $67 \pm 126$ & $-3.6 \pm 3.2$ & 0.1 & $-14 \pm 23$ & $18.1 \pm 0.3$ & $17.9 \pm 0.3$ & $10.75 \pm 0.03$ & $529 \pm 299$ & $518 \pm 293$ & $224 \pm 113$ \\
\hline & 1.408 & $7 \pm 278$ & $-2.8 \pm 0.1$ & 3.4 & $-21 \pm 1$ & $18.0 \pm 0.3$ & $17.9 \pm 0.2$ & $10.14 \pm 0.02$ & $525 \pm 297$ & $519 \pm 293$ & $206 \pm 102$ \\
\hline & 1.642 & $71 \pm 189$ & $-3.0 \pm 3.0$ & 0.6 & $-18 \pm 27$ & $17.5 \pm 0.4$ & $17.4 \pm 0.4$ & $9.72 \pm 0.03$ & $501 \pm 282$ & $494 \pm 278$ & $194 \pm 95$ \\
\hline \multirow[t]{5}{*}{ B1737+13 } & 0.408 & $84 \pm 109$ & $-4.2 \pm 0.8$ & 1.3 & $-14 \pm 4$ & $22.1 \pm 0.6$ & $18.2 \pm 0.8$ & - & $359 \pm 175$ & $252 \pm 122$ & - \\
\hline & 0.610 & $54 \pm 20$ & $-2.7 \pm 0.7$ & 5.2 & $-17 \pm 6$ & $28.4 \pm 1.5$ & $23.2 \pm 0.4$ & - & $581 \pm 293$ & $397 \pm 193$ & - \\
\hline & 0.910 & $85 \pm 58$ & $-2.4 \pm 0.2$ & 0.8 & $-24 \pm 3$ & $20.8 \pm 0.3$ & $20.1 \pm 0.3$ & $16.70 \pm 0.08$ & $322 \pm 155$ & $302 \pm 145$ & $216 \pm 101$ \\
\hline & 1.408 & $38 \pm 23$ & $-1.6 \pm 0.8$ & 2.4 & $-22 \pm 16$ & $24.6 \pm 0.6$ & $23.4 \pm 0.3$ & $15.88 \pm 0.08$ & $443 \pm 217$ & $401 \pm 195$ & $198 \pm 84$ \\
\hline & 1.642 & $32 \pm 58$ & $-1.5 \pm 2.3$ & 1.9 & $-21 \pm 47$ & $22.3 \pm 0.3$ & $21.5 \pm 0.3$ & $15.24 \pm 0.04$ & $367 \pm 177$ & $345 \pm 166$ & $184 \pm 92$ \\
\hline \multirow[t]{6}{*}{ В2045-16 } & 0.408 & $111 \pm 8$ & $-1.5 \pm 0.1$ & 0.5 & $-36 \pm 3$ & $19.4 \pm 0.4$ & $19.2 \pm 0.3$ & $12.88 \pm 0.01$ & $810 \pm 245$ & $792 \pm 239$ & $366 \pm 110$ \\
\hline & 0.606 & $129 \pm 2$ & $-1.4 \pm 0.1$ & 31.1 & $-32 \pm 1$ & $22.7 \pm 0.3$ & $22.3 \pm 0.4$ & $12.09 \pm 0.01$ & $1107 \pm 334$ & $1066 \pm 322$ & $325 \pm 97$ \\
\hline & 0.925 & $136 \pm 53$ & $-1.1 \pm 1.1$ & 0.5 & $-36 \pm 50$ & $17.8 \pm 0.4$ & $17.5 \pm 0.4$ & $11.28 \pm 0.02$ & $688 \pm 209$ & $661 \pm 200$ & $285 \pm 86$ \\
\hline & 1.408 & $121 \pm 6$ & $-1.4 \pm 0.1$ & 10.2 & $-35 \pm 3$ & $19.6 \pm 0.2$ & $19.3 \pm 0.2$ & $10.84 \pm 0.01$ & $829 \pm 250$ & $805 \pm 242$ & $264 \pm 80$ \\
\hline & 1.642 & $99 \pm 9$ & $-1.7 \pm 0.1$ & 4.3 & $-34 \pm 2$ & $18.8 \pm 0.3$ & $18.5 \pm 0.3$ & $10.71 \pm 0.01$ & $763 \pm 230$ & $737 \pm 222$ & $258 \pm 78$ \\
\hline & 4.850 & $92 \pm 77$ & $-2.4 \pm 0.3$ & 2.5 & $-24 \pm 4$ & $14.7 \pm 0.1$ & $14.3 \pm 0.1$ & $10.11 \pm 0.01$ & $471 \pm 141$ & $445 \pm 134$ & $232 \pm 70$ \\
\hline \multirow[t]{5}{*}{ B2111+46 } & 0.610 & $12 \pm 10$ & $-1.1 \pm 1.0$ & 15.8 & $-10 \pm 13$ & $88.7 \pm 1.3$ & $86.2 \pm 1.3$ & $59.07 \pm 0.02$ & $683 \pm 483$ & $647 \pm 456$ & $318 \pm 215$ \\
\hline & 0.925 & $13 \pm 25$ & $-1.4 \pm 2.8$ & 3.1 & $-9 \pm 25$ & $82.1 \pm 1.2$ & $79.1 \pm 1.1$ & $55.29 \pm 0.04$ & $591 \pm 414$ & $551 \pm 384$ & $281 \pm 189$ \\
\hline & 1.408 & $13 \pm 4$ & $-1.4 \pm 0.5$ & 61.2 & $-9 \pm 4$ & $86.9 \pm 1.9$ & $82.8 \pm 1.8$ & $51.12 \pm 0.02$ & $658 \pm 464$ & $601 \pm 422$ & $243 \pm 162$ \\
\hline & 1.642 & $14 \pm 28$ & $-1.5 \pm 3.0$ & 1.8 & $-9 \pm 26$ & $75.7 \pm 0.5$ & $74.5 \pm 0.4$ & $49.13 \pm 0.06$ & $507 \pm 352$ & $492 \pm 341$ & $225 \pm 149$ \\
\hline & 4.850 & $16 \pm 42$ & $-2.5 \pm 6.6$ & 1.1 & $-6 \pm 22$ & $75.1 \pm 0.6$ & $73.2 \pm 0.6$ & $46.07 \pm 0.03$ & $499 \pm 346$ & $476 \pm 330$ & $200 \pm 132$ \\
\hline
\end{tabular}

The beam radius $\rho$ appearing in Eq. (2) requires accurate knowledge of the viewing geometry $\alpha, \beta$ and the pulse width $W$. As we have discussed in the earlier section, even by using the most exhaustive information of multifrequency PPA traverses, exact determination of $\alpha$ and $\beta$ remains questionable due to the covarient nature of these parameters.
Nonetheless if we accept these values, then by measuring $W$ based on two defined points on the leading and the trailing edge of the profile $\rho, r_{\text {geo }}$ can be found. $r_{\text {geo }}$ corresponds to the emission height of these two defined points measured from the center of the neutron star provided we assume that the beam is circular and the two defined points on either side of the profile 

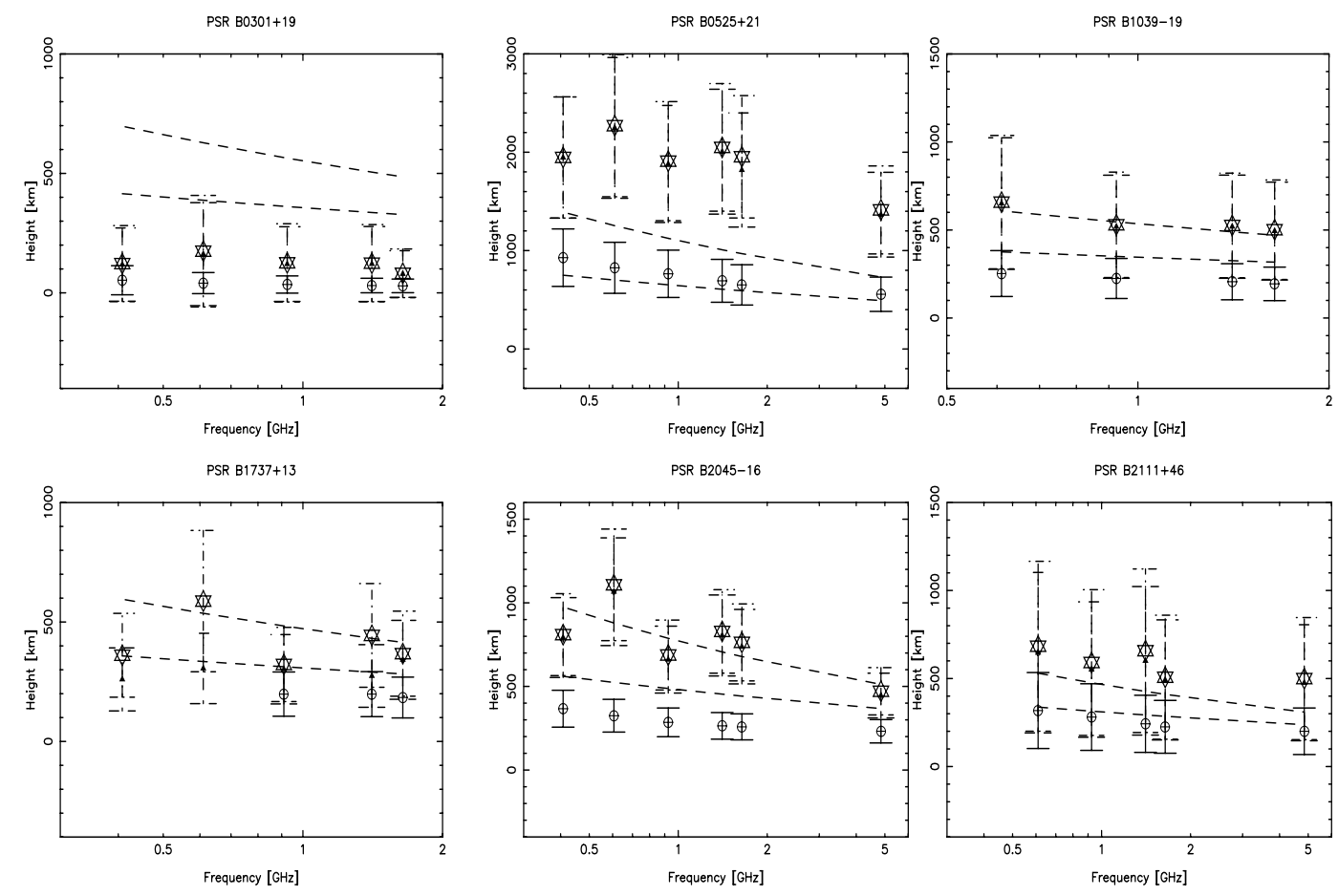

Fig. 2. The frequency dependence of $r_{\text {geo }}$ for PPCS (circles with crosses), OTS (stars) and OTHX (filled triangles) measurements. Here $r_{\text {geo }}$ calculated assumes $s=1$ for each case. The dashed line correspond to upper and lower limits of the $r_{\text {delay }}^{\mathrm{KG}}$ emission heights See text for further detail.

have the same emission height. The difficulty however arises in identifying these defined points. In the work of MR02 they noted that for the outer conal component $W$ measured using the outer peak to peak component separation (PPCS) is more stable with frequency compared to the outside half-power $(3 \mathrm{~dB})$ or outside 10\% profile width. Mitra \& Deshpande (1999) showed that the pulsar beam is consistant with a circular shape, however their analysis was based only on PPCS measurements. $\rho$ of the pulsar beam changes with frequencies and the defined outer edge points at different frequency corresponds to different dipolar field lines. Thus a stable variation of $\rho$ (or $W$ or $r_{\text {geo }}$ ) with frequency implies that the locus of the defined intensity points on these dipolar field lines follow a smoothly varying curve (not necessarily a circle). If $\rho$ obtained from two sets of such uniformly defined points (e.g. the peak to peak componet separation and the $10 \%$ widths) varies differently with frequency, it means that the intensity distribution of these two defined points are not concentric.

Here we measure profile widths based on three different definition (1) the PPCS, where we fit each profile with Gaussians to obtain the outer conal peaks accurately. (2) The outer three sigma (OTS) method, which involves choosing outer edges of the pulse profile at three times the off pulse rms level, and (3) using the outer three sigma method of HX97a (OTHX), where we first choose the outer three times rms point for the weaker component and then calculate the percentage level at that point with respect to the peak intensity of that component. Then we estimate the width by finding the location of the same percentage level with respect to the peak for the stronger component. For cases (2) and (3) an interpolation within the profile resolution was done to match the desired defined phases. The error in the phase $(\phi)$ for method (1) is obtained from the formal Gaussian fits while for cases (2) and (3) we use the relation suggested by HX97a namely,

$\sigma_{\phi}=\operatorname{rms} \frac{\tau}{\Delta I}$

where $\tau$ is the resolution of observation and $\Delta I$ is the gradient of the total intensity around the defined phase points. The measured widths and the errors are given in Table 2. For PSR B1737+17 the trailing component is seen to only appear clearly at frequencies 910, 1408 an $1642 \mathrm{MHz}$. Thus PPCS widths are reliable in these frequencies and only these values are quoted. Also the OTS and OTHX points are better measured at these frequencies.

Having chosen the outer points the crucial difficulty is to know the field line for these defined points with respect to the last open field line, i.e. the $s$ parameter. For outer PPCS measurements MR02 and GGb finds $s \sim 0.6$. Kijak \& Gil (1997) tried to overcome this problem by measuring $W$ based on outer conal edge phase points down to the lowest possible intensity level of about $1 \%$ and using $s=1$ for these points. They applied their method to a number of pulsars and found an empirical relation for emission height $r_{\mathrm{geo}}^{\mathrm{KG}}(\mathrm{km})=(400 \pm$ 80) $v_{\mathrm{GHz}}^{-0.26 \pm 0.09} \dot{P}_{15}^{0.07 \pm 0.03} P^{0.3 \pm 0.05}$, where $\dot{P}$ is the period derivative in units of $10^{-15} \mathrm{~s} / \mathrm{s}$ of the pulsar (Kijak \& Gil 2003). Since this relation is derived statistically using a large sample of pulsars, the errors introduced by the questionable viewing geometry is reduced. Nonetheless the unknown $s$ parameter does introduce uncertainty in estimating $r_{\text {geo }}$. 

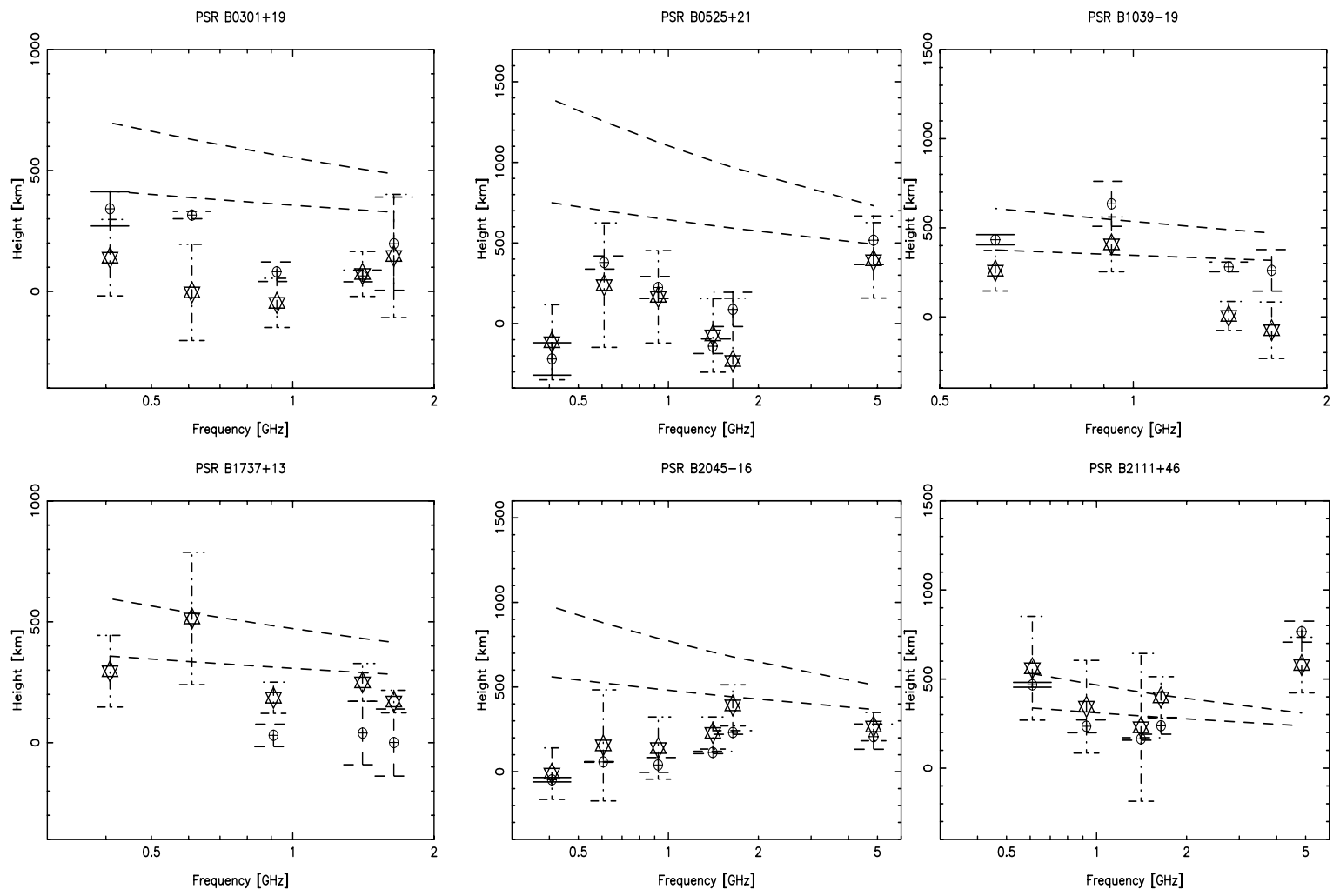

Fig. 3. The frequency dependence of $r_{\text {delay }}^{\mathrm{BCW}}$ for PPCS (circles with crosses) and OTS (stars) measurements. The dashed line corresponds to upper and lower limits of the $r_{\text {geo }}^{\mathrm{KG}}$ emission heights. See text for further details.

In Fig. 2 we plot $r_{\text {geo }}$ obtained by three different profile widths, and for comparison the upper and lower limit $r_{\text {geo }}^{\mathrm{KG}}$ relation is plotted as dashed lines. The following conclusions are apparent from Fig. 2.

(a) Emission heights obtained using PPCS decrease with increasing frequency in a rather stable manner. The OTS, OTHX together with the $10 \%$ and $3 \mathrm{~dB}$ widths noted by MR02 show more jittery behaviour with frequency. However the OTS and OTHX points are consistent with a stable variation within the error bars as seen in Fig. 3. High resolution and high quality signal to noise profiles are necessary to investigate this feature in detail.

(b) For five cases in our sample the OTS and OTHX emission heights are in good agreement with $r_{\text {geo }}^{\mathrm{KG}}$, given the error bars. Hence $r_{\mathrm{geo}}^{\mathrm{KG}}$ can be considered as robust upper limits for the emission height of the outer edge of the pulsar beam, although there might be deviations for individual cases. Only for the longest period pulsar PSR B $0525+21$ do we find that for OTS and OTHX measurements $r_{\text {geo }}>r_{\text {geo }}^{\mathrm{KG}}$. This can arise due to the fact that the empirical fit of $r_{\text {geo }}^{\mathrm{KG}}$ at the long period ( $>2 \mathrm{~s}$ ) end is not well constrained due to small number statistics.

Noticing that $r_{\text {geo }}$ measured using OTS and OTHX methods are in agreement within the error bars, we proceed only considering results corresponding to the OTS method since the results would be similar if OTHX methods were used.

\subsection{Delay emission heights: $r_{\text {delay }}$}

Based on the kinematical effects (A/R) BCW and GGa devised two independent ways to calculate delay emission heights $r_{\text {delay }}$. What makes the delay method attractive for measuring emission heights is that it is independent of the viewing geometry. Both the methods assume that the pulsar beam is symmetric about the fiducial phase. In this section we will discuss these two methods in succession and apply them to our sample of pulsars wherever possible.

The BCW method: this method involves measuring $\Delta \phi=$ $\phi_{\text {profile }}-\phi_{0}$ where $\phi_{\text {profile }}$ is the center of the pulse profile. Eventually the emission height is obtained using the formula,

$r_{\text {delay }}^{\mathrm{BCW}} \simeq-\frac{c}{4} \cdot \frac{\Delta \phi}{360^{\circ}} \cdot P \mathrm{~km}$.

Here $c=3 \times 10^{5} \mathrm{~km} \mathrm{~s}^{-1}$ is the velocity of light. The center of the pulse profile $\phi_{\text {profile }}$ requires accurate measurement of the phase $\phi_{\mathrm{t}}$ and $\phi_{1}$ at the extreme outer trailing and leading edge of the profile i.e., $\phi_{\text {profile }}=\phi_{t}+\left(\phi_{1}-\phi_{t}\right) / 2$. The phase $\phi_{0}$ is the fiducial phase which is considered to be the steepest gradient point of the PPA traverse. Note that $r_{\text {delay }}^{\mathrm{BCW}}$ corresponds to the actual emission height if it is assumed that the emission height corresponding to the trailing and leading edge $\left(r_{\text {edge }}\right)$ and the inner $\left(r_{\text {in }}\right)$ fiducial phase arises from the same height. To estimate $r_{\text {delay }}^{\mathrm{BCW}}$ one needs to use phase points corresponding to the lowest possible emission. In Fig. 3 we show the comparison of $r_{\text {delay }}^{\mathrm{BCW}}$ measured using PPCS and OTS measurements for all 
Table 3. Measurements of $\Delta \phi$ and the delay height $r_{\text {delay }}^{\mathrm{BCW}}$ estimates for the BCW methods corresponding to OTS and PPCS measurements. The geometrical height estimate of Kijak \& Gil (2003) is given as $r_{\text {geo }}^{\mathrm{KG}}$. The parameter $s=\left(\frac{r_{\text {geo }}^{\mathrm{Ors}}}{2 r_{\text {delay }}^{\mathrm{BCW}}}\right)^{0.5}$ is also listed (except the ones with negative $r_{\text {delay }}^{\mathrm{BCW}}$ values). See text for further details.

\begin{tabular}{|c|c|c|c|c|c|c|c|c|}
\hline PSR B & $\begin{array}{l}\text { Freq. } \\
(\mathrm{GHz})\end{array}$ & $\begin{array}{c}\Delta \phi_{\mathrm{OTS}} \\
\left(^{\circ}\right)\end{array}$ & $\begin{array}{c}\Delta \phi_{\mathrm{PPCS}} \\
\left(^{\circ}\right)\end{array}$ & $\begin{array}{c}r_{\text {delay }}^{\mathrm{BCW}}(\mathrm{OTS}) \\
(\mathrm{km})\end{array}$ & $\begin{array}{c}r_{\text {delay }}^{\mathrm{BCW}}(\mathrm{PPCS}) \\
(\mathrm{km})\end{array}$ & $\begin{array}{l}r_{\mathrm{geo}}^{\mathrm{KG}} \\
(\mathrm{km}) \\
\end{array}$ & $\frac{\left(r_{\text {geo }}^{\text {OTS }}\right)}{\left(r_{\text {delay }}^{\mathrm{BCW}}(\mathrm{OTS})\right)}$ & $s$ \\
\hline \multirow[t]{5}{*}{ B $0301+19$} & 0.408 & $-0.48 \pm 0.54$ & $-1.18 \pm 0.24$ & $139 \pm 157$ & $341 \pm 70$ & 567 & 0.87 & 0.66 \\
\hline & 0.610 & $0.01 \pm 0.68$ & $-1.09 \pm 0.05$ & $-4 \pm 198$ & $315 \pm 15$ & 511 & -42.71 & - \\
\hline & 0.925 & $0.16 \pm 0.35$ & $-0.28 \pm 0.13$ & $-47 \pm 101$ & $81 \pm 40$ & 458 & -2.64 & - \\
\hline & 1.408 & $-0.25 \pm 0.32$ & $-0.22 \pm 0.08$ & $72 \pm 92$ & $64 \pm 24$ & 411 & 1.71 & 0.92 \\
\hline & 1.642 & $-0.50 \pm 0.88$ & $-0.68 \pm 0.66$ & $146 \pm 254$ & $197 \pm 192$ & 395 & 0.55 & 0.52 \\
\hline \multirow[t]{6}{*}{ B $0525+21$} & 0.408 & $0.14 \pm 0.29$ & $0.28 \pm 0.12$ & $-115 \pm 232$ & $-219 \pm 100$ & 971 & -16.78 & - \\
\hline & 0.610 & $-0.30 \pm 0.49$ & $-0.48 \pm 0.05$ & $238 \pm 386$ & $378 \pm 40$ & 875 & 9.51 & 2.18 \\
\hline & 0.925 & $-0.21 \pm 0.36$ & $-0.28 \pm 0.08$ & $165 \pm 286$ & $224 \pm 68$ & 785 & 11.52 & 2.40 \\
\hline & 1.408 & $0.09 \pm 0.29$ & $0.18 \pm 0.05$ & $-73 \pm 228$ & $-140 \pm 45$ & 704 & -27.89 & - \\
\hline & 1.642 & $0.29 \pm 0.49$ & $-0.11 \pm 0.13$ & $-232 \pm 389$ & $87 \pm 106$ & 676 & -8.41 & - \\
\hline & 4.850 & $-0.50 \pm 0.29$ & $-0.66 \pm 0.19$ & $392 \pm 234$ & $516 \pm 150$ & 510 & 3.60 & 1.34 \\
\hline \multirow[t]{4}{*}{ B1039-19 } & 0.610 & $-0.89 \pm 0.39$ & $-1.49 \pm 0.09$ & $258 \pm 113$ & $432 \pm 28$ & 499 & 2.54 & 1.12 \\
\hline & 0.925 & $-1.40 \pm 0.53$ & $-2.19 \pm 0.43$ & $407 \pm 153$ & $634 \pm 126$ & 448 & 1.30 & 0.80 \\
\hline & 1.408 & $-0.01 \pm 0.28$ & $-0.97 \pm 0.09$ & $5 \pm 81$ & $280 \pm 27$ & 402 & 101.80 & 7.13 \\
\hline & 1.642 & $0.25 \pm 0.54$ & $-0.90 \pm 0.40$ & $-74 \pm 158$ & $261 \pm 116$ & 386 & -6.69 & - \\
\hline \multirow[t]{5}{*}{ B $1737+13$} & 0.408 & $-1.76 \pm 0.88$ & $-4.00 \pm 1.19$ & $295 \pm 148$ & - & 485 & 1.21 & 0.78 \\
\hline & 0.610 & $-3.07 \pm 1.63$ & $-4.10 \pm 0.90$ & $513 \pm 273$ & - & 437 & 1.10 & 0.74 \\
\hline & 0.910 & $-1.11 \pm 0.38$ & $-0.18 \pm 0.27$ & $185 \pm 64$ & $30 \pm 46$ & 394 & 1.73 & 0.93 \\
\hline & 1.408 & $-1.49 \pm 0.46$ & $-0.24 \pm 0.78$ & $249 \pm 78$ & $40 \pm 131$ & 351 & 1.79 & 0.94 \\
\hline & 1.642 & $-1.01 \pm 0.27$ & $-0.00 \pm 0.82$ & $170 \pm 46$ & $0 \pm 138$ & 337 & 2.15 & 1.03 \\
\hline \multirow[t]{6}{*}{ B2045-16 } & 0.408 & $0.02 \pm 0.37$ & $0.11 \pm 0.03$ & $-11 \pm 152$ & $-47 \pm 13$ & 730 & -70.41 & - \\
\hline & 0.606 & $-0.38 \pm 0.80$ & $-0.14 \pm 0.00$ & $155 \pm 328$ & $57 \pm 1$ & 659 & 7.12 & 1.88 \\
\hline & 0.925 & $-0.34 \pm 0.44$ & $-0.09 \pm 0.10$ & $139 \pm 183$ & $39 \pm 43$ & 590 & 4.92 & 1.56 \\
\hline & 1.408 & $-0.55 \pm 0.23$ & $-0.27 \pm 0.01$ & $228 \pm 94$ & $113 \pm 6$ & 529 & 3.62 & 1.34 \\
\hline & 1.642 & $-0.95 \pm 0.29$ & $-0.56 \pm 0.02$ & $391 \pm 121$ & $231 \pm 10$ & 508 & 1.94 & 0.98 \\
\hline & 4.850 & $-0.65 \pm 0.20$ & $-0.50 \pm 0.18$ & $266 \pm 83$ & $206 \pm 74$ & 384 & 1.76 & 0.94 \\
\hline \multirow[t]{5}{*}{ B $2111+46$} & 0.610 & $-2.65 \pm 1.37$ & $-2.21 \pm 0.06$ & $560 \pm 291$ & $467 \pm 13$ & 446 & 1.21 & 0.78 \\
\hline & 0.925 & $-1.62 \pm 1.23$ & $-1.10 \pm 0.16$ & $344 \pm 260$ & $234 \pm 35$ & 400 & 1.71 & 0.92 \\
\hline & 1.408 & $-1.08 \pm 1.96$ & $-0.77 \pm 0.03$ & $228 \pm 414$ & $163 \pm 7$ & 358 & 2.87 & 1.19 \\
\hline & 1.642 & $-1.87 \pm 0.55$ & $-1.12 \pm 0.22$ & $396 \pm 116$ & $237 \pm 46$ & 344 & 1.27 & 0.79 \\
\hline & 4.850 & $-2.73 \pm 0.73$ & $-3.62 \pm 0.27$ & $578 \pm 156$ & $765 \pm 58$ & 260 & 0.86 & 0.65 \\
\hline
\end{tabular}

our sample of pulsars as a function of frequency. We notice the following from the figure,

(a) the frequency dependence of $r_{\text {delay }}^{\mathrm{BCW}}$ with frequency is extremely erratic, in contrast to the behaviour of $r_{\text {geo }}$. This erratic behaviour is present for both PPCS and OTS methods. Note that in several cases $r_{\text {delay }}^{\mathrm{BCW}}$ has a negative value;

(b) $\triangle \phi$ measured using the PPCS and OTS methods gives values of $r_{\text {delay }}^{\mathrm{BCW}}$ that agree well within the error bars; 
(c) $r_{\text {delay }}^{\mathrm{BCW}}$ measured using OTS method is compatable with $r_{\text {delay }}^{\mathrm{BCW}}<r_{\text {geo }}^{\mathrm{KG}}$ or $r_{\text {geo }}^{\mathrm{OTS}}$ as seen from Tables 2 and 3 (except for the $4.85 \mathrm{GHz}$ point of PSR B2111+46).

To explain the erratic behaviour (point (a) above) of $r_{\text {delay }}^{\mathrm{BCW}}$ with frequency one first needs to critically assess the factors affecting determination of $\Delta \phi$ i.e. the phase points $\phi_{\mathrm{t}}, \phi_{1}$ and $\phi_{0}$. If the intensity distribution of the outer edge of the pulsar beam is irregular (or jittery), this will affect determination of $\phi_{\text {profile }}$. The reason is that $\phi_{\mathrm{t}}$ and $\phi_{1}$ points at various frequencies will no longer be symmetric with respect to the fiducial phase $\phi_{0}$ which is a breakdown of the key assumption of the BCW method. Conclusions drawn from the earlier sections suggest that PPCS, OTS and OTHX methods within the error bars agree with a smoothly varying profile edge. High quality observations are needed to discern this effect. Incorrect choice of the fiducial phase $\phi_{0}$ can also result in the observed erratic behaviour. However as mentioned in Sect. 3 we have beyond doubt identified the steepest gradient point as the fiducial phase for our sample of pulsars.

The uncertainties in the phase point measurements are in principle random and thus variation in the relation between $r_{\text {delay }}^{\mathrm{BCW}}$ and $r_{\text {geo }}$ should be observed. On the contrary the general tendency $r_{\text {delay }}^{\mathrm{BCW}}<r_{\text {geo }}^{\mathrm{KG}}$ (or $r_{\text {geo }}^{\mathrm{OTS}}$ ) is seen. Thus the obvious question is what other factors affect $r_{\text {delay }}^{\mathrm{BCW}}$ ? Here we discuss a few possibilities:

- Broad emission region: $\mathrm{BCW}$ in their pioneering work point out that their method gives $r_{\text {delay }}^{\mathrm{BCW}}$ averaged over all emission regions. Recently Dyks et al. (2003) provided a lucid description of this effect. They noted that $r_{\text {delay }}^{\mathrm{BCW}}$ can be decomposed in two parts where due to rotation the profile center shifts towards earlier phases by $-2 r_{\text {edge }} / R_{\mathrm{lc}}$ and the center of the PPA traverse is delayed by $2 r_{\mathrm{in}} / R_{\mathrm{lc}}$ (where $R_{\mathrm{lc}}$ is the radius of the light cylinder). For $r_{\text {edge }}=r_{\text {in }}$, the estimated emission height corresponds to the emission height of the outer conal edge. In the case $r_{\text {edge }} \gg r_{\text {in }}$ the height obtained is less than the outer edge emission, but can differ by a factor of 2 at most. We check this effect first by comparing $r_{\text {delay }}^{\mathrm{BCW}}$ obtained by the OTS method $r_{\text {geo }}^{\text {OTS }}$. The value $r_{\text {geo }}^{\text {OTS }} / r_{\text {delay }}^{\text {OTS }}$ (listed in Col. 8 of Table 3 ) shows a large spread and for several cases is greater than 2 . Given this variety no simple conclusion can be drawn regarding the relation between the inner and outer edge emission.

The difficulty in the above comparison is that the $s$ factor corresponding to the OTS points is unknown. If we assume $r_{\text {edge }} \gg r_{\text {in }}$, then we can equate $r_{\text {geo }}^{\text {OTS }} \mathrm{s}^{-2}=2 r_{\text {delay }}^{\text {OTS }}$ and find the $s$ parameter. In Table 3 the $s$ parameter is given and a wide variety of $s$ values are seen thus making it difficult to reach any meaningful conclusion. However $r_{\text {delay }}^{\text {OTS }}$ can be affected by several other effects of unknown magnitude which makes it impossible to extract the $s$ parameter.

- Magnetic field sweepback (MFSB): magnetospheric rotation and currents can give rise to magnetic field distortions at sufficiently high emission altitude $r$. The effect acts in the direction opposite to the stellar rotation. Unfortunately the magnitude of this effect is not clearly understood. Shitov (1983) estimate the effect to be of the order of $\left(r / R_{\mathrm{lc}}\right)^{3}$ while
Arendt \& Eilek (1998) find it to be proportional to $\left(r / R_{\mathrm{lc}}\right)$. Using more sophisticated calculations Dyks et al. (2003) show that this effect is as important as $\mathrm{A} / \mathrm{R}$ i.e. of the order of $x\left(r / R_{\mathrm{lc}}\right)$, where $x$ is close to unity, confirming results obtained by Arendt \& Eilek (1998). Their calculations demonstrate that this effect can cause distortions in the outer edge of the beam which further depend on the viewing geometry.

Phase shifts due to MFSB and aberration effects act in opposite directions. As a result, an increase in $\Delta \phi$ due to $\mathrm{A} / \mathrm{R}$ is cancelled by a decrease in the phase shift due to MFSB. This underestimates $\Delta \phi$ and hence $r_{\text {delay }}$ and thus can explain why $r_{\text {delay }}<r_{\text {geo }}^{\text {OTS }}$. Further, the numerical calculations of Dyks et al. (2003) show that the magnitude of the MFSB effect varies for the outer edge of the radio beam as a function of $\beta$ or different magnetic field lines corresponding to the outer edge. Thus for multifrequency observations since the outer edges corresponds to different field lines, MFSB effects can be frequency-dependent.

If we assume that all the emission arises from the same height, and only $\mathrm{A} / \mathrm{R}$ and the MFSB effect contribute to $\Delta \phi$, we can decompose $\Delta \phi=\Delta \phi_{\mathrm{A} / \mathrm{R}}-\Delta \phi_{\mathrm{MFSB}}$. If we claim that $r_{\text {delay }}=$ $r_{\text {geo }}^{\mathrm{KG}}$, then $\Delta \phi_{\mathrm{MFSB}}$ should be added to the observed $\Delta \phi$ to satisfy the equality. This requires $\Delta \phi_{\mathrm{MFSB}}=x\left(4 \times 360^{\circ} \times c \times r_{\mathrm{geo}}^{\mathrm{KG}} / P\right)$, where the factor $x$ is not known. For $x \sim 1$ one can in principle get fairly good agreement with the delay and the geometrical heights as seen in Fig. 4. If the MFSB effect is indeed operating in pulsars then we have found observational confirmation of this for the first time.

Given the multitude of effects of unknown magnitude simultaneously affecting $r_{\text {delay }}^{\mathrm{BCW}}$, the height estimates obtained from this method are affected by a factor of a few.

The GGa method: this method is also based on the A/R effect suggesting an alternative way of deriving delay emission heights based on total intensity profiles. The method applies to pulsars with both core and conal emission. Here the fiducial phase is assumed to be the peak of the core emission. The emission heights obtained are heights with respect to the height of the core emission $\left(r_{\text {core }}\right)$.

GGa's method involves measuring the phase shift $\Delta \phi$ between the center obtained from the PPCS and the peak of the core emission. GGa connects $\Delta \phi$ to the emission radius as $r_{\text {delay }}^{\mathrm{GGa}}=(c / 2)\left(P / 360^{\circ}\right) \Delta \phi /(1+\sin \alpha)$. An important correction to this formula has been suggested by Dyks et al. (2003) where they find

$r_{\text {delay }}^{\mathrm{GGa}}=-\frac{c}{2} \frac{\Delta \phi}{360^{\circ}} P \mathrm{~km}$.

Note that the modified formula is independent of the emission geometry. GGa and GGb applied their method to seven M type pulsars at $325 \mathrm{MHz}$. They demonstrated that the centers of both outer and the inner emission components lead the peak of the core emission supporting the $\mathrm{A} / \mathrm{R}$ picture. They also found $\Delta \phi$ for the inner components are systematically smaller than the outer components, suggesting that the inner components arise closer to the stellar surface than the outer components and the core emission lies below the conal emission and closest to the stellar surface. Their observation supports the picture that the pulsar emission beam has nested cones of emission 

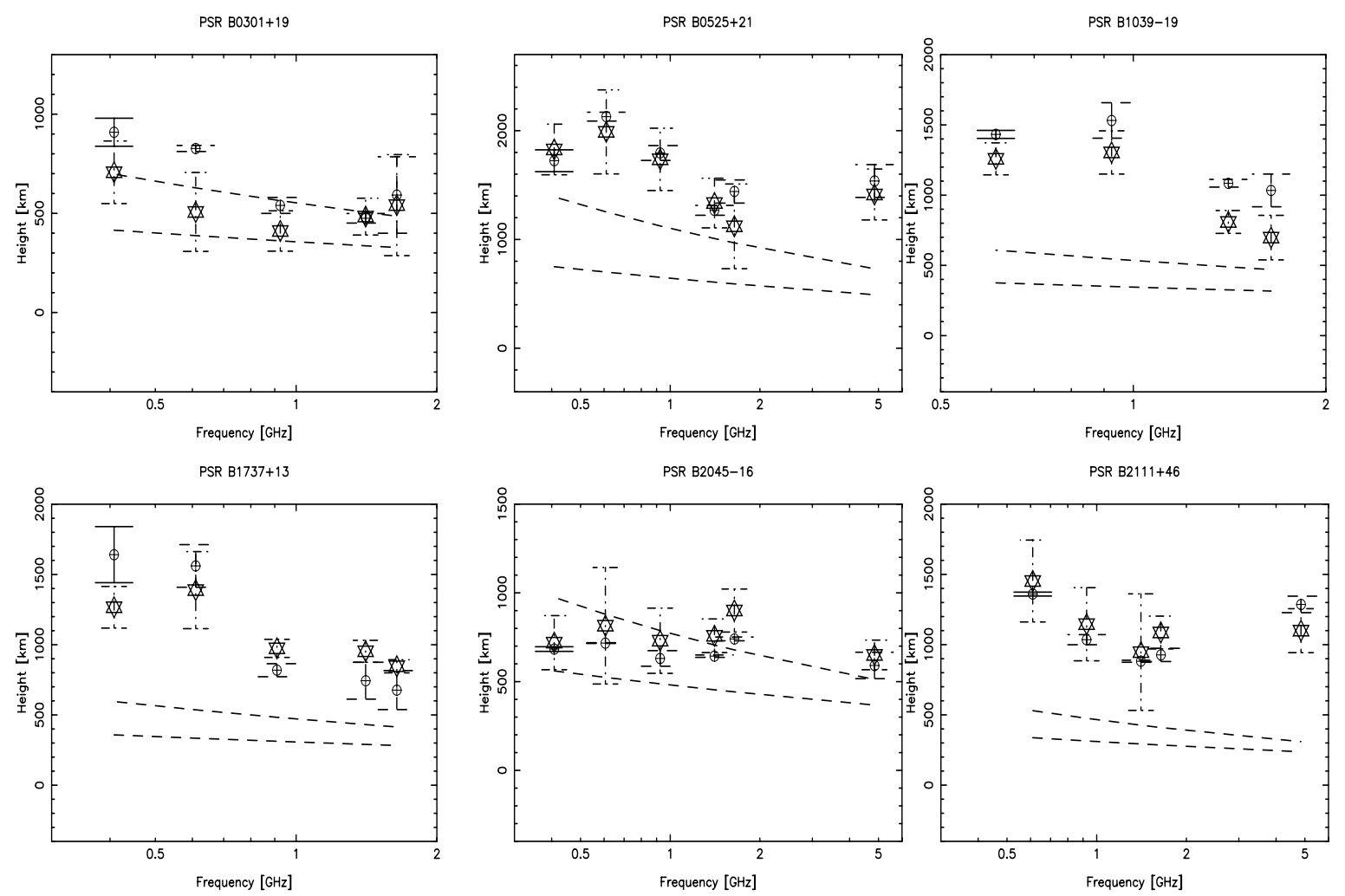

Fig. 4. The frequency dependence of $r_{\text {delay }}^{\mathrm{BCW}}$ corrected for a simulated MFSB effect for PPCS (circles with crosses) and OTS (stars) measurements. The dashed line corresponds to upper and lower limits of the $r_{\text {geo }}^{\mathrm{KG}}$ emission heights. See text for further details.

(e.g. Mitra \& Deshpande 1999) with the inner cones arising at lower heights compared to the outer cones as was suggested by Rankin (1983).

Considering the above scenario if $r_{\text {core }}=R_{*}$, a straightforward comparison can be made between $r_{\text {delay }}^{\mathrm{GGa}}$ and $r_{\text {geo }}$. If $r_{\text {delay }}^{\mathrm{GGa}} \sim$ $r_{\text {geo }}$, then one can conclude that the field line corresponding to OTS points are the last open field lines such that $s=1$. In the case $r_{\text {delay }}^{\mathrm{GGa}}>r_{\text {geo }}$ the OTS points correspond to $s<1$, giving lower values of $r_{\text {geo }}^{\mathrm{OTS}}$. Thus claiming that $r_{\text {delay }}^{\mathrm{GGa}}$ should be equal to $r_{\text {geo }}^{\mathrm{OTS}}$ and using Eq. (2) one can find $s=\left(r_{\text {geo }}^{\mathrm{OTS}} / r_{\text {delay }}^{\mathrm{GGa}}\right)^{0.5}$. We use PSR B2045-16 and PSR B2111+46 in our data set where such a comparison is possible. In Table 4 values for $r_{\text {delay }}^{\mathrm{GGa}}$ and the $s$ parameter for these two pulsars are given. In Fig. $5 r_{\text {delay }}^{\mathrm{GGa}}$ is shown. For both the cases one observes that

(a) $r_{\text {delay }}^{\mathrm{GGa}}>r_{\text {geo }}^{\mathrm{OTS}}$ and $s<1$ as given in Table 4;

(b) $r_{\text {delay }}^{\mathrm{GGa}}$ obtained by PPCS and OTS methods agrees within the error bars;

(c) variation of $r_{\text {delay }}^{\mathrm{GGa}}$ with frequency is erratic. For PSR B2045-16 the variation is different from that obtained by the BCW method;

(d) for both the cases $r_{\text {delay }}^{\mathrm{GGa}}>r_{\text {delay }}^{\mathrm{BCW}}$.

A crucial assumption in the GGa method is considering the peak of the core emission as the fiducial phase containing the dipole axis, the rotation axis and the observer's line of sight. GGa already mentions that if $r_{\text {core }}$ originates higher than the polar cap, then the height of the core needs to be added to $r_{\text {delay }}^{\mathrm{GGa}}$ to obtain correct radio emission heights. Even in this case the peak of the core emission needs to be the fiducial phase as defined above.

In Sect. 3 based on our combined PPA traverses we have firmly concluded that the steepest gradient point in the PPA traverse serves as the fiducial phase in our sample of pulsars. Thus for the core to originate from the polar cap and the peak of the core to be the fiducial phase it should be coincident with the steepest gradient point. Here we notice that for PSR B2045-16 and PSR B2111+46 even by eye inspection (see Fig. 1 for examples) it is clearly seen that the center of the core lags behind the steepest gradient of the combined PPA traverse. This feature is also evident in PSR B1737+13 at frequencies of 408 and $610 \mathrm{MHz}$ where the core component is clearly identifiable. In Fig. 6 we show in the top panel the alignment of PSR B2045-16 with respect to the core emission. The core emission clearly lags the steepest gradient point of the PPA traverse shown in the lower panel and the amount of lag is seen to increase with decreasing frequency.

The central core emission is thought to originate from the surface of the polar cap (Rankin 1990, 1993). Rankin noticed that the $3 \mathrm{~dB}$ core widths at $1 \mathrm{GHz}$ for six interpulsars depend on the pulsar period and $\alpha$ and this relates extremely well to the size of the polar cap estimated using dipolar field lines. It is hence conjectured that the core emission is consistent with emission arising from a filled polar cap with a bivariant Gaussian intensity distribution, and the peak of the core lies 
Table 4. Measurements of $\Delta \phi$ and the delay height $r_{\text {delay }}^{\mathrm{GGa}}$ estimates for the BCW methods corresponding to OTS and PPCS measurements. The geometrical height estimate of Kijak \& Gil (2003) is given as $r_{\mathrm{geo}}^{\mathrm{KG}}$. The parameter $s=\left(\frac{r_{\mathrm{geo}}^{\mathrm{OTS}}}{2 r_{\text {delay }}^{\mathrm{GGa}}}\right)^{0.5}$ is also listed. See text for further details.

\begin{tabular}{|c|c|c|c|c|c|c|c|c|}
\hline PSR B & $\begin{array}{l}\text { Freq. } \\
(\mathrm{GHz})\end{array}$ & $\begin{array}{c}\Delta \phi_{\text {OTS }} \\
\left(^{\circ}\right)\end{array}$ & $\begin{array}{c}\Delta \phi_{\mathrm{PPCS}} \\
\left(^{\circ}\right)\end{array}$ & $\begin{array}{c}r_{\text {delay }}^{\mathrm{GGa}}(\mathrm{OTS}) \\
(\mathrm{km})\end{array}$ & $\begin{array}{c}r_{\text {delay }}^{\mathrm{GGa}}(\mathrm{PPCS}) \\
(\mathrm{km})\end{array}$ & $\begin{array}{l}r_{\mathrm{geo}}^{\mathrm{KG}} \\
(\mathrm{km})\end{array}$ & $\frac{\left(r_{\text {geo }}^{\text {OTS }}\right)}{\left(r_{\text {delay }}^{\mathrm{GGa}}(O T S)\right)}$ & $S$ \\
\hline \multirow[t]{5}{*}{ B2045-16 } & 0.408 & $-2.41 \pm 0.35$ & $-2.32 \pm 0.03$ & $1970 \pm 287$ & $1898 \pm 25$ & 730 & 0.41 & 0.64 \\
\hline & 0.606 & $-2.29 \pm 0.36$ & $-2.20 \pm 0.00$ & $1873 \pm 298$ & $1803 \pm 3$ & 659 & 0.57 & 0.75 \\
\hline & 0.925 & $-2.31 \pm 0.46$ & $-2.06 \pm 0.10$ & $1890 \pm 382$ & $1689 \pm 87$ & 590 & 0.36 & 0.60 \\
\hline & 1.408 & $-2.21 \pm 0.24$ & $-1.94 \pm 0.01$ & $1814 \pm 201$ & $1592 \pm 13$ & 529 & 0.45 & 0.67 \\
\hline & 1.642 & $-2.47 \pm 0.37$ & $-2.08 \pm 0.02$ & $2026 \pm 305$ & $1704 \pm 21$ & 508 & 0.37 & 0.61 \\
\hline \multirow[t]{5}{*}{ B2111+46 } & 0.610 & $-3.31 \pm 1.30$ & $-2.97 \pm 0.06$ & $1403 \pm 549$ & $1259 \pm 27$ & 446 & 0.49 & 0.70 \\
\hline & 0.925 & $-2.61 \pm 1.24$ & $-2.10 \pm 0.16$ & $1105 \pm 525$ & $891 \pm 71$ & 400 & 0.53 & 0.76 \\
\hline & 1.408 & $-2.60 \pm 1.42$ & $-2.47 \pm 0.03$ & $1100 \pm 601$ & $1045 \pm 14$ & 358 & 0.60 & 0.78 \\
\hline & 1.642 & $-3.57 \pm 0.55$ & $-2.82 \pm 0.22$ & $1512 \pm 235$ & $1195 \pm 94$ & 344 & 0.33 & 0.57 \\
\hline & 4.850 & $-3.12 \pm 1.29$ & $-3.35 \pm 0.27$ & $1322 \pm 545$ & $1417 \pm 117$ & 260 & 0.39 & 0.62 \\
\hline
\end{tabular}
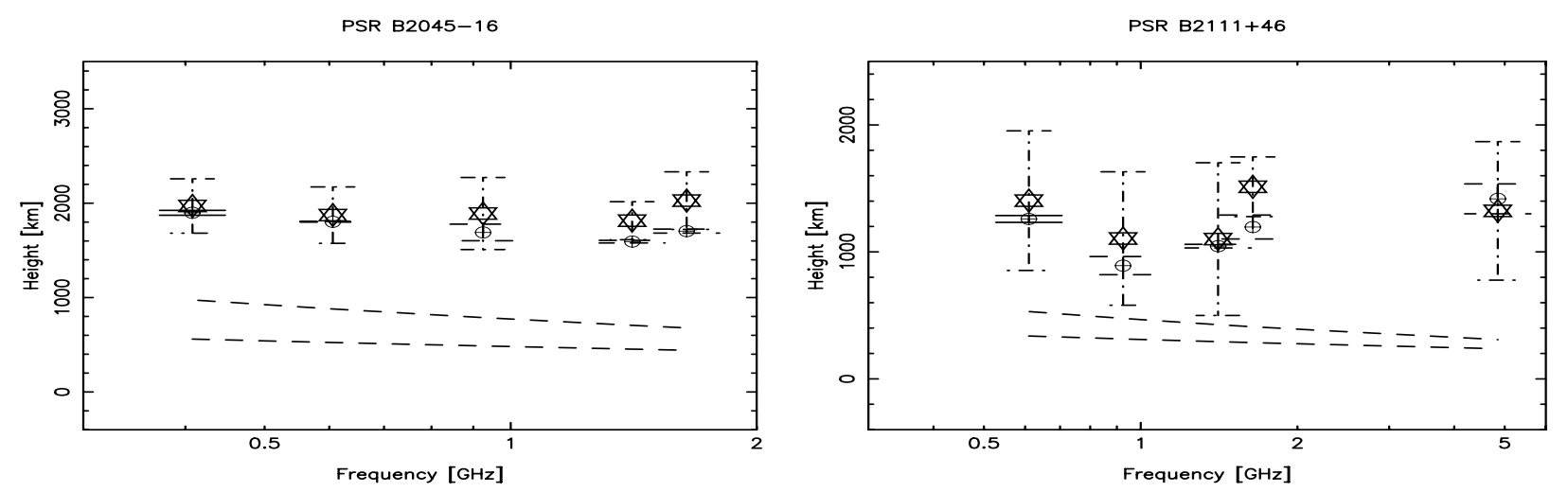

Fig. 5. The frequency dependence of $r_{\text {delay }}^{\mathrm{GGa}}$ for PPCS (circles with crosses) and OTS (stars) measurements. The dashed line corresponds to upper and lower limits of the $r_{\text {geo }}^{\mathrm{KG}}$ emission heights See text for further details.

in the fiducial plane containing the dipolar magnetic axis. If the core emission arises from the polar cap then: (1) according to the BCW model the phase offset between the core peak and the steepest gradient of the PPA traverse is $(4 / c) R_{*}\left(360^{\circ} / P\right)$. For a typical $1 \mathrm{~s}$ pulsar and $R_{*}=10 \mathrm{~km}$ this number is extremely small $\sim 0.05^{\circ}$, typically a factor of 10 less than the typical resolution of observations. On the other hand the observed lag of the core emission with respect to the steepest gradient point is in disagreement with the core emission arising from the polar cap. (2) If the core emission arises from the polar cap at all frequencies the width of the core should be frequencyindependent. However, as seen in Fig. 6 the core width evolves significantly with frequency. (3) The line-of-sight $\beta$ should be smaller than the polar cap radius $\rho_{\mathrm{pc}}\left({ }^{\circ}\right)=1.24 R_{*} P^{-0.5} / 10$. In Table 1 values of $\rho_{\mathrm{pc}}$ are given (for $R_{*}=10 \pm 3 \mathrm{~km}$ ) and for the core pulsars $\rho_{\mathrm{pc}} \sim \beta$ indicating that the line of sight is almost grazing the polar cap rather than central cuts. Dyks et al. (2003) found $\rho_{\mathrm{pc}}>\beta$ for the core-dominated pulsar PSR B0450-18. This evidence hints that the core emission does not originate from the polar cap surface and the peak of the core is not the fiducial phase. Breakdown of this assumption introduces unknown uncertainties in $r_{\text {delay }}^{\mathrm{GGa}}$ and thus comparison with other emission height estimates are difficult ${ }^{2}$.

It is however puzzling that if the peak of the core is not the fiducial phase why the seven pulsar studied by GGa and GGb have the core lagging the profile center. Lyne \& Manchester (1988) already noted that they find 58 coredominated pulsars for which " 35 lead the profile center and 23 trail it". Perhaps this observational feature needs to be reviewed before we can reach a firm conclusion. It should be remembered the MFSB effect as discussed earlier also affects determination of $r_{\text {delay }}^{\mathrm{GGa}}$ in a very similar fashion.

2 Note that we have based our arguments accepting that by combining the PPA traverse and establishing its striking agreement with the RVM, we have found the fiducial phase as the steepest gradient point. Any other explanation refuting the steepest gradient point to be the fiducial phase will affect the conclusion drawn in this paper. 
PSR B2045-16

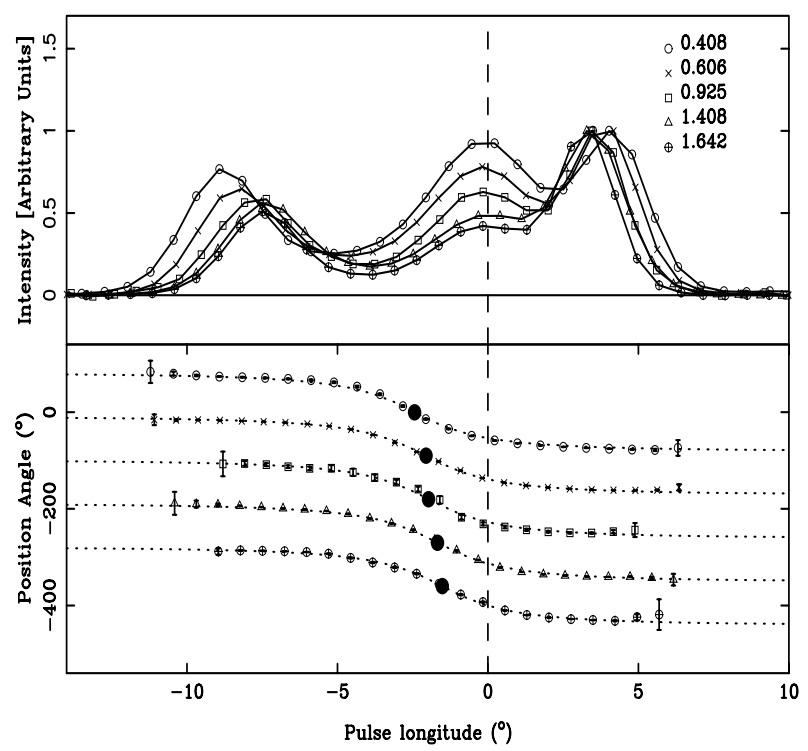

Fig. 6. The multifrequency alignment of PSR B2045-16 based on the peak of the core component. The top panel shows the intensity and the bottom panel the PPA traverse. The dark dots in the bottom panel corresponds to the steepest gradient point in the PPA traverse (see text for details).

\subsection{Effect of propagation on emission heights}

Propagation effects within the pulsar magnetosphere can influence polarization and total intensity properties in pulsars as was advocated by Barnard \& Arons (1986) and recently reinvestigated in a series of works by Petrova \& Lyubarskii (2000, 2003) and references therein. The effect of propagation can complicate estimation of $r_{\text {geo }}$. This arises in the form of refractive effects acting on the outer conal components. Refraction changes the direction of the rays arising from the last open field lines. This affects the widths of the outer conal component in a frequency-dependent manner and is prominent at higher frequencies. The effect has been considered as an explanation for saturation of pulse widths (see MR02) observed at high frequencies (Mckinnon 1997). However the magnitude of this effect depends on the plasma parameters in the pulsar magnetosphere which are difficult to constrain. However as a consequence the width $W$ is larger and $r_{\text {geo }}$ corresponds to emission heights that are higher than the actual emission heights.

The effect of propagation can also affect determination of $r_{\text {delay }}$. Unfortunately the theory for such effects is still not well understood. Grossly it is conjectured that in the course of propagation through the plasma flow the PPA follows the direction of the local magnetic field up to a distance $r_{\mathrm{p}}$, higher than the total intensity emission. According to the $\mathrm{BCW}$ model the effect will tend to increase $\Delta \phi$, since the the steepest gradient will be delayed by a larger amount compared to the total intensity profile. This should however result in higher estimates of $r_{\text {delay }}$ compared to $r_{\text {geo }}$, contrary to what is observed. Stongly varying $r_{\mathrm{p}}$ across the profile can complicate interpretation of $r_{\text {delay }}$ significantly. This can also affect phase determination of the beam edge in a frequency-dependent manner, explaining the erratic nature of $r_{\text {delay }}$.

Further PPAs are influenced mostly in regions in the pulse where the amount of circular polarization is high. A general feature observed in average profiles is that cores are more circularly polarized than the cones, and thus PPAs will tend to be more affected near the core component. Single pulse analyses have recently shown that circular polarization is a property of both cone and core pulsars (Karastergiou et al. 2003). The averaging effect washes out the circular polarization in the cones, however retains it in the central core emission. Thus on average profiles for outer cones propagation effects might be averaged out, but can still influence the core component. This can influence determination of $\phi_{0}$ and consequently $r_{\text {delay }}$. The remarkable similarity of the PPA traverses over frequency seems to rule out any major propagation effect that might influence PPA traverses in our sample of pulsars.

\section{Summary}

Important conclusions drawn in this paper are:

(a) For the first time using six bright conal pulsars we construct the combined PPA traverse ranging from frequencies between $\sim 400 \mathrm{MHz}$ and $\sim 5 \mathrm{GHz}$. The combined PPA traverse is in excellent agreement with the RVM confirming that radio emission arises from regions with perfectly dipolar magnetic field.

(b) The fiducial phase in a pulse is chosen to be the point lying in the plane containing the dipolar magnetic axis, the rotaion axis and the observers line of sight. Due to the retardation effect $(\mathrm{BCW})$ and RFM this fiducial plane changes with frequency. By combining the PPA traverses we eliminate this frequency-dependent effect and construct a common fiducial plane containing the fiducial phase (see dark dots in the bottom panel of Fig. 6 or/and dashed vertical lines in Fig. 1). Then noting the striking similarity to the RVM we conclude that the steepest gradient point of the combined PPA traverse in the fiducial phase for our sample of pulsars. This phase thus should be treated as the fiducial phase for the application of cold plasma dedispersion.

(c) We find in general $r_{\text {delay }}^{\mathrm{BCW}}<r_{\text {geo }}^{\mathrm{OTS}}$ and the frequency dependence of $r_{\text {delay }}^{\mathrm{BCW}}$ is more erratic than $r_{\mathrm{geo}}^{\mathrm{OTS}}$. The former effect can be understood by considering a broad emission region (this means emission at any given frequency arises over a range of heights in the pulsar magnetosphere) and/or the MFSB effect recently proposed by Dyks et al. (2003). The latter effect can arise due to an irregular (or jittery) shape of the edge of the pulsar beam.

(d) The peak of the core emission for three pulsars is seen to lag the steepest gradient point of the PPA traverse over several frequencies. The width of the core is seen to increase with decreasing frequency. This strongly suggests that the core emission emanates higher than the polar cap surface and the peak emission does not lie in the fiducial plane containing the dipole axis, rotation axis and the line of sight.

To understand issues relating to pulse emission height's future studies producing combined PPA traverses using lower 
frequency observations $(<400 \mathrm{MHz})$ should enable us to get better constraints on the viewing geometry. On the theoretical side there is a great need to develop and understand the details of the MFSB effect. Futher efforts should be made to understand the observed lag between the steepest gradient point and the peak of the core emission which has been highlighted in great detail in this work.

Acknowledgements. X. H. Li thanks Prof. G. J. Qiao and X. H. Sun for valuable discussion. We thank J. Gil, S. Petrova, J. H. Seiradakis, M. Kramer, D. Athanasiadis, Y. Gupta, J. Dyks and J. Kijak for insightful discussions and comments on the manuscript. We thank J. L. Han, A. Jessner \& R. Wielebinski for constant encouragement during this work and several useful comments. We thank J. Rankin for critical comments on this work which helped us to improve the manuscript substantially. The project is supported by the National Natural Science Foundation of China (19903003 and 10025313), the National Key Basic Research Science Foundation of China (G1990754) and the partner group of MPIFR at NAOC.

\section{References}

Arendt, P. N., \& Eilek, J. A. 1998, ApJ, submitted [arXiv: astro-ph/9801257]

Athanasiadis, D., Li, X. H., Kramer, M., \& Mitra, D., in preparation Backer, D., \& Rankin, J. 1980, ApJS, 42, 143

Barnard, J. J., \& Arons, J. 1986, ApJ, 302, 138

Blaskiewicz, M., Cordes, J. M., \& Wasserman, I. 1991, ApJ, 370, 643 (BCW)

Cordes, J. M. 1978, ApJ, 222, 1006

Dyks, J., Rudak, B., \& Harding, A. K. 2003, ApJ, submitted [arXiv: astro-ph/0307251]

Everett, J. E., \& Weisberg, J. M. 2001, ApJ, 553, 341 (EW)

Gangadhara, R. T., \& Gupta, Y. 2001, ApJ, 555, 31 (GGa)

Gil, J. A., Gronkowski, P., \& Rudnicki, W. 1984, A\&A, 132, 312

Gil, J. A. 1991, A\&A, 243, 219

Gil, J., \& Krawczyk, A. 1997, MNRAS, 285, 561

Gil, J. A., \& Sendyk, M. 2000, ApJ, 541, 351

Goldreich, P., \& Julian, W. H. 1969, ApJ, 157, 869

Gould, D. M., \& Lyne, A. G. 1998, MNRAS, 301, 235
Gupta, Y., \& Gangadhara, R. T. 2003, ApJ, 584, 418 (GGb)

Han, J. L., Manchester, R. N., Xu, R. X., \& Qiao, G. J. 1998, MNRAS, 300,373

Hibschman, J. A., \& Arons, J. 2001, ApJ, 546, 382

von Hoensbroech, A., \& Xilouris, K. M. 1997, A\&AS, 126, 121 (HX97a)

von Hoensbroech, A., \& Xilouris, K. M. 1997, A\&A, 324, 981 (HX97b)

Karastergiou, A., Kramer, M., Johnston, S., et al. 2002, A\&A, 391, 247

Karastergiou, A., Johnston, S., Mitra, D., van Leeuwen, A. G. J., \& Edwards, R. 2003, MNRAS, 344L, 69K

Kijak, J., \& Gil, J. 1997, MNRAS, 288, 631

Kijak, J., \& Gil, J. 1998, MNRAS, 299, 855

Kijak, J., \& Gil, J. 2003, A\&A, 397, 969

Kramer, M. 1994, A\&AS, 107, 527

Li, X. H., \& Han, J. L. 2003, A\&A, 410, 253

Lyne, A. G., \& Manchester, R. N. 1988, MNRAS, 234, 477

Malov, I. F., \& Suleimanova, S. A. 1998, Astron. Rep., 42, 388

Manchester, R. N., \& Taylor, J. H. 1977 (San Francisco: W. H. Freeman), 36

Marquart, D. W. 1963, J. Soc. Ind. Apll. Math., 11, 431

Mckinnon, M. M. 1997, ApJ, 475, 763

Mitra, D., \& Deshpande, A. A. 1999, A\&A, 346, 906

Mitra, D., \& Rankin, J. M. 2002, ApJ, 577, 322 (MR02)

Narayan, R., \& Vivekanand, M. 1982, A\&A, 113, L3

Petrova, S. A., \& Lyubarskii, Y. E. 2000, A\&A, 355, 1168

Petrova, S. A. 2000, A\&A, 360, 592

Petrova, S. A. 2003, A\&A, 408, 1057

Press, W. H., Flannery, B. P., \& Teukolsky, S. A. 1986 (Cambridge: University Press)

Radhakrishnan, V., \& Cooke, D. J. 1969, Astrophys. Lett., 3, 225

Rankin, J. M. 1983, ApJ, 274, 333

Rankin, J. M. 1990, ApJ, 352, 247

Rankin, J. M. 1993a, ApJ, 405, 285 (Rankin 1993a)

Rankin, J. M. 1993b, ApJS, 85, 145 (Rankin 1993b)

Ruderman, M. A., \& Sutherland, P. G. 1975, ApJ, 196, 51

Shitov, Y. P. 1983, SvA, 27, 314

Stinebring, D. R., Cordes, J. M., Rankin, J. M., Weisberg, J. M., \& Boriakoff, V. 1984, ApJS, 55, 247 